\title{
Secrecy capacities of compound quantum wiretap channels and applications
}

\author{
Holger Boche, ${ }^{1}$ Minglai Cai, ${ }^{1}$ Ning Cai, ${ }^{2}$ and Christian Deppe ${ }^{1}$ \\ ${ }^{1}$ Lehrstuhl für Theoretische Informationstechnik, Technische Universität München, München, German\}团 \\ ${ }^{2}$ The State Key Laboratory of Integrated Services Networks, University of Xidian, Xian, Chind
}

\begin{abstract}
We determine the secrecy capacity of the compound channel with quantum wiretapper and channel state information at the transmitter. Moreover, we derive a lower bound on the secrecy capacity of this channel without channel state information and determine the secrecy capacity of the compound classical-quantum wiretap channel with channel state information at the transmitter. We use this result to derive a proof for a lower bound on the entanglement generating capacity of the compound quantum channel. We also derive a proof for the formula for entanglement generating capacity of the compound quantum channel with channel state information at the encoder which was given in additional information (cf. I. Bjelaković, H. Boche, and J. Nötzel, Proceedings of International Symposium on Information Theory ISIT, 1889-1893, Korea, 2009).
\end{abstract}

Keywords: Compound channel; Wiretap channels; Quantum channels; Entanglement generation

\section{INTRODUCTION}

Our goal is to analyze information transmission over a set of indexed channels, which is called a compound channel. The indices are referred to as channel states. Only one channel in this set is actually used for the information transmission, but the users can not control which channel in the set will be used. The capacity of the classical compound channel was determined in [14].

A compound channel with an eavesdropper is called a compound wiretap channel. We define a compound wiretap channel as a family of pairs of channels $\left\{\left(\mathrm{W}_{t}, \mathrm{~V}_{t}\right)\right.$ : $t=1, \ldots, T\}$ with a common input alphabet and possibly different output alphabets, connecting a sender with two receivers, a legal one and a wiretapper, where $t$ stands for the state of the channel pair $\left(\mathrm{W}_{t}, \mathrm{~V}_{t}\right)$. The legitimate receiver accesses the output of the first channel $W_{t}$ in the pair $\left(\mathrm{W}_{t}, \mathrm{~V}_{t}\right)$, and the wiretapper observes the output of the second part $\mathrm{V}_{t}$ in the pair $\left(\mathrm{W}_{t}, \mathrm{~V}_{t}\right)$, respectively, when a state $t$ governs the channel. A code for the channel conveys information to the legal receiver such that the wiretapper's knowledge of the transmitted information can be kept arbitrarily small. This is a generalization of Wyner's classical wiretap channel [42] to a case of multiple channel states. In [42], the author required that the wiretapper can not detect the message using a weak security criterion (cf. Remark 2). For the achievable secrecy rate, we use the worst-case interpretation, i.e., it is limited by the secrecy rate when the destination has the worst channel state.

We deal with two communication scenarios. In the first one, only the sender is informed about the index $t$, or in other words, he has CSI, where CSI is an abbreviation for "channel state information". In the second one, both the sender and the receiver do not have any information about that index at all.

\footnotetext{
* [boche, minglai.cai, christian.deppe]@tum.de

$\dagger$ caining@mail.xidian.edu.cn
}

The classical compound wiretap channels were introduced in [28]. A lower bound on the classical secrecy capacity was obtained under the condition that the sender does not have any knowledge about the CSI. In [28], the authors required that the receiver's average error goes to zero and that the wiretapper is not able to detect the message, with respect to the same security criterion as in [42]. The result of [28] was improved in [13] by using a stronger condition for the limit of the legitimate receiver's error, i.e., the maximal error should go to zero, as well as a stronger condition for the security criterion (c.f. Remark 2). Furthermore, the secrecy capacity was determined for the case in which the sender had knowledge about the CSI.

In this paper, we consider quantum channels. A quantum channel can transmit both classical and quantum information. We consider the capacity of quantum channels carrying classical information. This is equivalent to considering the capacity of classical-quantum channels, where the classical-quantum channels are quantum channels whose sender's inputs are classical variables. The classical capacity of quantum channels has been determined in [23], 24], 34], and 35]. In general, there are two ways to represent a quantum channel with linear algebraic tools (cf. e.g. Section VII), either as a sum of several transformations, or as a single unitary transformation which explicitly includes the unobserved environment. We use the latter one for our result in the entanglement generating capacity. These two representations can both be used to determine the entanglement generating capacity for quantum channels, but it is unknown if this holds for the entanglement generating capacity of compound quantum channels.

We analyze two variants of compound wiretap quantum channels in this paper. The first variant is called the classical compound channel with quantum wiretapper. In this channel model, we assume that the wiretap channels are quantum channels, while the legal transmission channels are classical channels. The second variant is called the compound classical-quantum wiretap channel. In this channel model, we assume that both fami- 
lies of channels are quantum channels, while the sender transmits classical information.

A quantum wiretap channel is described by a map $N$ which maps the set of density operators on a system $G^{\mathfrak{A}}$ to the set of density operators on a composite system $G^{\mathfrak{B} Z}$. Here, $G^{\mathfrak{A}}$ is the the system of the sender, $G^{\mathfrak{B}}$ is the system observed by the legal receiver, and $G^{\mathfrak{Z}}$ is the system observed by the wiretapper. We only allow passive eavesdropping attacks, i.e., the actions which the wiretapper performs on his system have no influence on the legal receiver's system. For active eavesdropping attacks, i.e., the actions which the wiretapper performs on his system have influences on the legal receiver's system, c.f. please arbitrarily varying quantum channels, which are generalizations of compound quantum channels ([2], [10], [15], [1], and [17]). Following [19], we define a quantum wiretap channel with passive eavesdropping attacks as a pair of channels $(W, V)$. For every density operator $\zeta$ on $G^{\mathfrak{A}}$ we define $W(\zeta):=\operatorname{tr}_{\mathfrak{B}}(N(\zeta))$ and $V(\zeta):=\operatorname{tr}_{\mathfrak{Z}}(N(\zeta))$.

References [8] and [7] are two well-known examples for secure quantum information transmission using quantum key distributions. Good one-shot results for quantum channels with a wiretapper who is limited in his actions have been obtained. But our goal is to have a more general theory for channel security in quantum information theory, i.e. message transmission should be secure against every possible kind of eavesdropping. Furthermore, we are interested in asymptotic behavior when we deliver a large volume of messages by many channel uses. Therefore, we consider an alternate paradigm for the design of quantum channel systems, which is called embedded security. Here, we embed protocols with a guaranteed security into the bottom layerof the model of communications systems, which is the physical layer. We use channels, for example, fiber-optic cables, for generating and transmitting secure messages which generate secure keys, or secure message transmissions.

Since we allow every possible kind of eavesdropping, we use the Holevo $\chi$ quantity as our security criterion (cf. (13)). By [24] and [35] the wiretapper can never obtain more information asymptotically than the Holevo $\chi$ quantity, no matter which strategy the wiretapper uses. Another widely used security criterion is the variational distance between $p^{A} p^{Z}$ and $p^{A Z}$. Here $p^{A Z}$ is the joint probability describing the sender's random variable and the wiretapper's random variable. $p^{A}$ is the marginal probability describing the sender's random variable and $p^{Z}$ is the marginal probability describing the wiretapper's random variable, respectively. The Holevo $\chi$ quantity using a strong condition for the security criterion (c.f. Remark 21) is stronger than the variational distance between $p^{A} p^{Z}$ and $p^{A Z}$ in the sense that if the Holevo $\chi$ quantity between $p^{A}$ and the wiretap channel's output (using strong condition) goes to zero, the variational distance between $p^{A} p^{Z}$ and $p^{A Z}$ goes to zero as well (cf.
[21] and [16]).

Our results are summarized as follows. Under the condition that the sender has knowledge about the CSI, the secrecy capacity for these two channel models is derived. Additionally, when the sender does not have any knowledge about the CSI, we determine the secrecy capacity of the compound classical-quantum wiretap channel, and give a lower bound for the secrecy capacity of the classical compound channel with quantum wiretapper.

As an application of the above results, we turn to the question: "What is the maximal amount of entanglement that we can generate or transmit over a given compound quantum channel?" For the sender and the receiver, the objective is to share a nearly maximally entangled state on a $\left(2^{n R} \times 2^{n R}\right)$ dimensional Hilbert space by using a large number $n$ instances of the compound quantum channel. In [5] it is shown how to send a large amount of entangled quantum states through a noisy quantum channel such that the channel does not modify the entanglement. However, the study of entanglement generation allows a noisy quantum channel to modify the entanglement, as long as the transmitters can use a recovery algorithm to restore the entanglement. The entanglement generating capacity of a quantum channel has been determined in [20] and [29]. The entanglement generating capacities of a compound quantum channel with and without CSI have been determined in [11] and [12]. In our paper we derive a lower bound on the entanglement generating capacity of the compound quantum channel by using an alternative technique to the method in [11] and [12] (cf. SectionVII). Furthermore, we derive the entanglement generating capacity of the compound quantum channel with CSI at the encoder using an alternative technique.

The main definitions are given in Section III

In Section III, we present some known results for the classical compound wiretap channel which are used for the proof of the results in Section IV.

In Section IV, we discuss the classical compound channel with a quantum wiretapper. For the case when the sender has the full knowledge about the CSI, we derive the secrecy capacity. For the case when the sender does not know the CSI, we give a lower bound for the secrecy capacity. In this channel model the wiretapper uses classical-quantum channels.

In Section V] we derive the secrecy capacity of the compound classical-quantum wiretap channel with CSI. In this model both the receiver and the wiretapper use classical quantum channels and the set of the channel states may be finite or infinite.

In Section VI, we use the results of Section $\nabla$ to derive a lower bound on the entanglement generating capacity for the compound quantum channel. The entanglement generating capacity of the compound quantum channel with CSI at the encoder is also derived.

In Section VII, we discuss our proof of the previous section and remind the reader about the two ways to 
represent a quantum channel with linear algebraic tools.

\section{PRELIMINARIES}

For a finite set $B$, we denote the set of probability distributions on $B$ by $P(B)$. Let $\rho_{1}$ and $\rho_{2}$ be Hermitian operators on a finite-dimensional complex Hilbert space $G$. We say $\rho_{1} \geq \rho_{2}$ and $\rho_{2} \leq \rho_{1}$ if $\rho_{1}-\rho_{2}$ is positivesemidefinite. For a finite-dimensional complex Hilbert space $G$, we denote the set of density operators on $G$ by

$\mathcal{S}(G):=\left\{\rho \in \mathcal{L}(G): \rho\right.$ is Hermitian, $\left.\rho \geq 0_{G}, \operatorname{tr}(\rho)=1\right\}$,

where $\mathcal{L}(G)$ is the set of linear operators on $G$, and $0_{G}$ is the null matrix on $G$. Note that any operator in $\mathcal{S}(G)$ is bounded.

For finite sets $A$ and $B$, we define a (discrete) classical channel $\mathrm{V}: A \rightarrow P(B), A \ni x \rightarrow \mathrm{V}(x) \in P(B)$ to be a system characterized by a probability transition matrix $\mathrm{V}(\cdot \mid \cdot)$. For $x \in A$ and $y \in B, \mathrm{~V}(y \mid x)$ expresses the probability of the output symbol $y$ when we send the symbol $x$ through the channel. The channel is said to be memoryless if the probability distribution of the output depends only on the input at that time and is conditionally independent of previous channel inputs and outputs.

Let $n \in \mathbb{N}$. For a finite set $A$ we define $A^{n}:=$ $\left\{\left(a_{1}, \ldots, a_{n}\right): a_{i} \in A \forall i \in\{1, \ldots, n\}\right\}$. For a finite set $A$ and a finite-dimensional complex Hilbert space $H$, the space which the vector $\left\{v_{1} \otimes \cdots \otimes v_{n}: v_{i} \in H \forall i \in\right.$ $\{1, \ldots, n\}\}$ span is defined by $H^{\otimes n}$. We also write $a^{n}$ and $v^{n}$ for the elements of $A^{n}$ and $H^{\otimes n}$, respectively.

Let $n \in \mathbb{N}$. For a discrete random variable $X$ on a finite set $A$, and a discrete random variable $Y$ on a finite set $B$, we denote the Shannon entropy by $H(X)$ and the mutual information between $X$ and $Y$ by $I(X ; Y)$ (cf. [40]).

For a probability distribution $P$ on a finite set $A$, a conditional stochastic matrix $\Lambda$, and a positive constant $\delta$, we denote the set of typical sequences by $\mathcal{T}_{P, \delta}^{n}$ and the set of conditionally typical sequences by $\mathcal{T}_{\Lambda, \delta}^{n}\left(x^{n}\right)$ (here we use the strong condition) (cf. [40]).

For finite-dimensional complex Hilbert spaces $G$ and $G^{\prime}$, a quantum channel $V: \mathcal{S}(G) \rightarrow \mathcal{S}\left(G^{\prime}\right), \mathcal{S}(G) \ni$ $\rho \rightarrow V(\rho) \in \mathcal{S}\left(G^{\prime}\right)$ is represented by a completely positive trace preserving map, which accepts input quantum states in $\mathcal{S}(G)$ and produces output quantum states in $\mathcal{S}\left(G^{\prime}\right)$.

If the sender wants to transmit a classical message $m \in M$ to the receiver using a quantum channel, his encoding procedure will include a classical-to-quantum encoder $M \rightarrow \mathcal{S}(G)$ to prepare a quantum message state $\rho \in \mathcal{S}(G)$ suitable as an input for the channel. If the sender's encoding is restricted to transmitting an indexed finite set of quantum states $\left\{\rho_{x}: x \in A\right\} \subset \mathcal{S}(G)$, then we can consider the choice of the signal quantum states $\rho_{x}$ to be a component of the channel. Thus, we obtain a channel with classical inputs $x \in A$ and quantum outputs:
$\sigma_{x}:=V\left(\rho_{x}\right)$, which we call a classical-quantum channel. This is a map V: $A \rightarrow \mathcal{S}\left(G^{\prime}\right), \mathfrak{X} \ni x \rightarrow \mathrm{V}(x) \in \mathcal{S}\left(G^{\prime}\right)$ which is represented by the set of $|A|$ possible output quantum states $\left\{\sigma_{x}=\mathrm{V}(x):=V\left(\rho_{x}\right): x \in A\right\} \subset \mathcal{S}\left(G^{\prime}\right)$, meaning that each classical input of $x \in A$ leads to a distinct quantum output $\sigma_{x} \in \mathcal{S}\left(G^{\prime}\right)$.

Let $n \in \mathbb{N}$. Following [40] we define the $n$-th memoryless extension of the stochastic matrix $\mathrm{V}$ by $\mathrm{V}^{n}$, i.e., for $x^{n}=\left(x_{1}, \ldots, x_{n}\right) \in A^{n}$ and $y^{n}=\left(y_{1}, \ldots, y_{n}\right) \in B^{n}$, $\mathrm{V}^{n}\left(y^{n} \mid x^{n}\right)=\prod_{i=1}^{n} \mathrm{~V}\left(y_{i} \mid x_{i}\right)$. Following [40], we define the $n$-th extension of quantum channel and classicalquantum channel as follows. Associated with $V$ and $\mathrm{V}$ are the channel maps on an $n$-block $V^{\otimes n}: \mathcal{S}\left(G^{\otimes n}\right) \rightarrow$ $\mathcal{S}\left(G^{\prime \otimes n}\right)$ and $\mathrm{V}^{\otimes n}: A^{n} \rightarrow \mathcal{S}\left(G^{\prime \otimes n}\right)$, such that for any $\rho^{n}=\rho_{1} \otimes \cdots \otimes \rho_{n} \in \mathcal{S}\left(G^{\otimes n}\right)$ and any $x^{n}=\left(x_{1}, \ldots, x_{n}\right) \in$ $A^{n}, V^{\otimes n}\left(\rho^{n}\right)=V\left(\rho_{1}\right) \otimes \cdots \otimes V\left(\rho_{n}\right)$, and $\mathrm{V}_{t}^{\otimes n}\left(x^{n}\right)=$ $\mathrm{V}\left(x_{1}\right) \otimes \cdots \otimes \mathrm{V}\left(x_{n}\right)$, respectively. Although the outcomes of $V^{\otimes n}$ can be written as $n$ tuples, we still regard them as elements of $\mathcal{S}\left(G^{\otimes n}\right)$ because for the proof of our results, we need tools which are defined on the space $G^{\otimes n}$ (c.f. (11)-(7) .

Let $A$ be a finite set and $G$ be a finite-dimensional complex Hilbert space. For a quantum state $\rho \in \mathcal{S}(G)$, we denote the von Neumann entropy of $\rho$ by

$$
S(\rho)=-\operatorname{tr}(\rho \log \rho) .
$$

Let V: $A \rightarrow \mathcal{S}(G)$ be a classical-quantum channel. Following [3], for $P \in P(A)$ the conditional entropy of the channel for $\mathrm{V}$ with input distribution $P$ is denoted by

$$
S(\mathrm{~V} \mid P):=\sum_{x \in A} P(x) S(\mathrm{~V}(x)) .
$$

Remark 1 The following definition is a more general definition of conditional entropy in quantum information theory. Let $\mathfrak{P}$ and $\mathfrak{Q}$ be quantum systems. We denote the Hilbert space of $\mathfrak{P}$ and $\mathfrak{Q}$ by $G^{\mathfrak{P}}$ and $G^{\mathfrak{Q}}$, respectively. Let $\phi^{\mathfrak{P Q}}$ be a bipartite quantum state in $\mathcal{S}\left(G^{\mathfrak{P Q}}\right)$. We denote $S(\mathfrak{P} \mid \mathfrak{Q})_{\rho}:=S\left(\phi^{\mathfrak{P Q}}\right)-S\left(\phi^{\mathfrak{Q}}\right)$. Here $\phi^{\mathfrak{Q}}=\operatorname{tr}_{\mathfrak{P}}\left(\phi^{\mathfrak{P Q}}\right)$.

For quantum states $\rho$ and $\sigma \in \mathcal{S}(G)$, we denote the fidelity of $\rho$ and $\sigma$ by

$$
F(\rho, \sigma):=\|\sqrt{\rho} \sqrt{\sigma}\|_{1}^{2},
$$

where $\|\cdot\|_{1}$ stands for the trace norm.

We denote the identity operator on a space $G$ by $\mathrm{I}_{G}$ and the identity superoperator on $G$ by $\operatorname{id}_{G}$.

Let $A$ be a finite set and let $G$ and $G^{\prime}$ be a finitedimensional complex Hilbert spaces. For a quantum state $\rho \in \mathcal{S}(G)$ and a quantum channel $V: \mathcal{S}(G) \rightarrow \mathcal{S}\left(G^{\prime}\right)$ the coherent information is defined as

$$
I_{C}(\rho, V):=S(V(\rho))-S\left(\left(\mathrm{I}_{G} \otimes V\right)(|\psi\rangle\langle\psi|)\right),
$$

where $|\psi\rangle\langle\psi|$ is an arbitrary purification of $\rho$ in $\mathcal{S}(G) \otimes$ $\mathcal{S}(G)$. Let $\Phi:=\left\{\rho_{x}: x \in A\right\}$ be a set of quantum states 
labeled by elements of $A$. For a probability distribution $P$ on $A$ the Holevo $\chi$ quantity is defined as

$$
\chi(P ; \Phi):=S\left(\sum_{x \in A} P(x) \rho_{x}\right)-\sum_{x \in A} P(x) S\left(\rho_{x}\right) .
$$

Let $n \in \mathbb{N}$, let $A$ be a finite set, and $G$ be a finitedimensional complex Hilbert space. For $\rho \in \mathcal{S}(G)$ and $\alpha>0$ there exists an orthogonal subspace projector $\Pi_{\rho, \alpha}$ commuting with $\rho^{n}$ and satisfying

$$
\begin{gathered}
\operatorname{tr}\left(\rho^{n} \Pi_{\rho, \alpha}\right) \geq 1-\frac{d}{4 n \alpha^{2}}, \\
\operatorname{tr}\left(\Pi_{\rho, \alpha}\right) \leq 2^{n S(\rho)+K d \alpha \sqrt{n}}, \\
\Pi_{\rho, \alpha} \cdot \rho^{n} \cdot \Pi_{\rho, \alpha} \leq 2^{-n S(\rho)+K d \alpha \sqrt{n}} \Pi_{\rho, \alpha},
\end{gathered}
$$

where $d:=\operatorname{dim} H$, and $K$ is a positive constant.

Let V: $A \rightarrow \mathcal{S}(G)$ be a classical-quantum channel. For $P \in P(A), \alpha>0$ and $x^{n} \in A^{n}$ there exists an orthogonal subspace projector $\Pi_{\mathrm{V}, \alpha}\left(x^{n}\right)$ commuting with $\mathrm{V}^{\otimes n}\left(x^{n}\right)$ and satisfying

$$
\begin{gathered}
\operatorname{tr}\left(\mathrm{V}^{\otimes n}\left(x^{n}\right) \Pi_{\mathrm{V}, \alpha}\left(x^{n}\right)\right) \geq 1-\frac{a d}{4 n \alpha^{2}}, \\
\operatorname{tr}\left(\Pi_{\mathrm{V}, \alpha}\left(x^{n}\right)\right) \leq 2^{n S(\mathrm{v} \mid P)+K a d \alpha \sqrt{n}}, \\
\Pi_{\mathrm{V}, \alpha}\left(x^{n}\right) \cdot \mathrm{V}^{\otimes n}\left(x^{n}\right) \cdot \Pi_{\mathrm{V}, \alpha}\left(x^{n}\right) \\
\quad \leq 2^{-n S(\mathrm{~V} \mid P)+K a d \alpha \sqrt{n}} \Pi_{\mathrm{V}, \alpha}\left(x^{n}\right),
\end{gathered}
$$

where $a:=|\{A\}|$, and $K$ is a positive constant (cf. [40]).

For the classical-quantum channel V : $A \rightarrow \mathcal{S}(G)$, every probability distribution $P$ on $A$ defines a quantum state $P$ V on $\mathcal{S}(G)$, which is the resulting quantum state at the output of $\mathrm{V}$ when the input is sent according to $P$. Thus for $\alpha^{\prime}>0$ we can define an orthogonal subspace projector $\Pi_{P \mathrm{v}, \alpha^{\prime} \sqrt{a}}$ which fulfills (11), (2), and (3) (here we set $\rho=P \mathrm{~V}$ and $\alpha=\alpha^{\prime} \sqrt{a}$ ). Furthermore, for $\Pi_{P \mathrm{v}, \alpha^{\prime} \sqrt{a}}$ we have the following inequality:

$$
\operatorname{tr}\left(\mathrm{V}^{\otimes n}\left(x^{n}\right) \cdot \Pi_{P \mathrm{~V}, \alpha \sqrt{a}}\right) \geq 1-\frac{a d}{4 n \alpha^{2}} .
$$

Let $A, B$, and $C$ be finite sets, $H, H^{\prime}$, and $H^{\prime \prime}$ be complex Hilbert spaces, and $\mathfrak{P}$ and $\mathfrak{Q}$ be quantum systems. We denote the Hilbert space of $\mathfrak{P}$ and $\mathfrak{Q}$ by $H^{\mathfrak{P}}$ and $H^{\mathfrak{Q}}$, respectively. Let $\theta:=\{1, \ldots, T\}$ be a finite set. For every $t \in \theta$ let

$\mathrm{W}_{t}$ be a classical channel $A \rightarrow P(B)$;

$\vee_{t}$ be a classical channel $A \rightarrow P(C)$;

$\mathrm{V}_{t}$ be a classical-quantum channel $A \rightarrow \mathcal{S}(H)$;

$W_{t}$ be a quantum channel $\mathcal{S}\left(H^{\prime}\right) \rightarrow \mathcal{S}\left(H^{\prime \prime}\right)$;
$V_{t}$ be a quantum channel $\mathcal{S}\left(H^{\prime}\right) \rightarrow \mathcal{S}(H)$;

$N_{t}$ be a quantum channel $\mathcal{S}\left(H^{\mathfrak{P}}\right) \rightarrow \mathcal{S}\left(H^{\mathfrak{Q}}\right)$.

We call the set of the classical channel pairs $\left(\mathrm{W}_{t}, \mathrm{~V}_{t}\right)_{t \in \theta}$ a (classical) compound wiretap channel. When the channel state is $t$, and the sender inputs $x \in A$ into the channel, the receiver receives the output $y \in B$ with probability $\mathrm{W}_{t}(y \mid x)$, while the wiretapper receives the output $z \in Z$ with probability $\mathrm{V}_{t}(z \mid x)$.

We call the set of the classical channel and classicalquantum channel pairs $\left(\mathrm{W}_{t}, \mathrm{~V}_{t}\right)_{t \in \theta}$ a compound channel with quantum wiretapper. When the channel state is $t$ and the sender inputs $x \in A$ into the channel, the receiver receives the output $y \in B$ with probability $\mathrm{W}_{t}(y \mid x)$, while the wiretapper receives an output quantum state $\mathrm{V}_{t}^{\otimes n}(x) \in \mathcal{S}(H)$.

We call the set of the quantum channel pairs $\left(W_{t}, V_{t}\right)_{t \in \theta}$ a quantum compound wiretap channel. When the channel state is $t$ and the sender inputs a quantum state $\rho \in \mathcal{S}\left(H^{\prime}\right)$ into the channel, the receiver receives an output quantum state $W_{t}(\rho) \in \mathcal{S}\left(H^{\prime \prime}\right)$, while the wiretapper receives an output quantum state $V_{t}(\rho) \in \mathcal{S}(H)$.

We call the set of the quantum channel $\left(N_{t}\right)_{t \in \theta}$ a quantum compound channel. When the channel state is $t$ and the sender inputs a quantum state $\rho^{\mathfrak{P}} \in$ $\mathcal{S}\left(H^{\mathfrak{P}}\right)$ into the channel, the receiver receives an output quantum state $N_{t}\left(\rho^{\mathfrak{P}}\right) \in \mathcal{S}\left(H^{\mathfrak{Q}}\right)$.

We distinguish two different scenarios according to the sender's knowledge of the channel state:

- the sender has the CSI, i.e. he knows which $t$ the channel state actually is,

- the sender does not have any CSI.

In both cases we assume that the receiver does not have any CSI, but the wiretapper always has the full knowledge of the CSI. Of course we also have the case where both the sender and the receiver have the CSI, but this case is equivalent to the case when we only have one pair of channels $\left(W_{t}, V_{t}\right)$, instead of a family of pairs of channels $\left\{\left(W_{t}, V_{t}\right): t=1, \ldots, T\right\}$.

An $\left(n, J_{n}\right)$ code for the classical compound wiretap channel $\left(\mathrm{W}_{t}, \mathrm{~V}_{t}\right)_{t \in \theta}$ consists of a stochastic encoder $E$ : $\left\{1, \ldots, J_{n}\right\} \rightarrow P\left(A^{n}\right)$ specified by a matrix of conditional probabilities $E(\cdot \mid \cdot)$, and a collection of mutually disjoint sets $\left\{D_{j} \subset B^{n}: j \in\left\{1, \ldots, J_{n}\right\}\right\}$ (decoding sets).

If the sender has the CSI, then instead of using a single code for all channel states, we may use the following strategy. For every $t \in \theta$, the sender and the receiver build an $\left(n, J_{n}\right)$ code $\left(E_{t},\left\{D_{j}: j=1, \ldots, J_{n}\right\}\right)$ such that all codes in $\left\{\left(E_{t},\left\{D_{j}: j=1, \ldots, J_{n}\right\}\right): t \in \theta\right\}$ share the same decoding sets $\left\{D_{j}: j=1, \ldots, J_{n}\right\}$, which do not depend on $t$, to transform the message.

A non-negative number $R$ is an achievable secrecy rate 
for the classical compound wiretap channel $\left(\mathrm{W}_{t}, \mathrm{~V}_{t}\right)$ having CSI at the encoder, if for every positive $\varepsilon, \delta$, every $t \in \theta$, and a sufficiently large $n$ there is an $\left(n, J_{n}\right)$ code $\left(E_{t},\left\{D_{j}: j=1, \ldots, J_{n}\right\}\right)$, such that $\frac{1}{n} \log J_{n} \geq R-\delta$, and

$$
\begin{gathered}
\max _{t \in \theta} \max _{j \in\left\{1, \ldots, J_{n}\right\}} \sum_{x^{n} \in A^{n}} E_{t}\left(x^{n} \mid j\right) \mathrm{W}_{t}^{n}\left(D_{j}^{c} \mid x^{n}\right) \leq \varepsilon, \\
\max _{t \in \theta} I\left(X_{\text {uni }} ; K_{t}^{n}\right) \leq \varepsilon,
\end{gathered}
$$

where $X_{u n i}$ is a random variable uniformly distributed on $\left\{1, \ldots, J_{n}\right\} . K_{t}^{n}$ are the resulting random variables at the output of wiretap channels $\mathrm{V}_{t}^{n}$. Here we denote the complement of a set $\Xi$ by $\Xi^{c}$.

Remark $2 A$ weaker and widely used security criterion, e.g. in [28] (also cf. [42] for wiretap channel's security criterion), is obtained if we replace (9) with $\max _{t \in \theta} \frac{1}{n} I\left(X_{\text {uni }} ; K_{t}^{n}\right) \leq \varepsilon$. In this paper we will follow [15] and use (9).

A non-negative number $R$ is an achievable secrecy rate for the classical compound wiretap channel $\left(\mathrm{W}_{t}, \mathrm{~V}_{t}\right)$ having no CSI at the encoder, if for every positive $\varepsilon$, $\delta$ and a sufficiently large $n$ there is an $\left(n, J_{n}\right)$ code $\left(E,\left\{D_{j}: j=1, \ldots, J_{n}\right\}\right)$ such that $\frac{1}{n} \log J_{n} \geq R-\delta$, and

$$
\begin{gathered}
\max _{t \in \theta} \max _{j \in\left\{1, \ldots, J_{n}\right\}} \sum_{x^{n} \in A^{n}} E\left(x^{n} \mid j\right) \mathrm{W}_{t}^{n}\left(D_{j}^{c} \mid x^{n}\right) \leq \varepsilon, \\
\max _{t \in \theta} I\left(X_{\text {uni }} ; K_{t}^{n}\right) \leq \varepsilon .
\end{gathered}
$$

An $\left(n, J_{n}\right)$ code for the compound channel with quantum wiretapper $\left(\mathrm{W}_{t}, \mathrm{~V}_{t}\right)_{t \in \theta}$ consists of a stochastic encoder $E:\left\{1, \ldots, J_{n}\right\} \rightarrow P\left(A^{n}\right)$ and a collection of mutually disjoint sets $\left\{D_{j} \subset B^{n}: j \in\left\{1, \ldots, J_{n}\right\}\right\}$ (decoding sets).

A non-negative number $R$ is an achievable secrecy rate for the compound channel with quantum wiretapper $\left(\mathrm{W}_{t}, \mathrm{~V}_{t}\right)_{t \in \theta}$ having CSI at the encoder, if for every positive $\varepsilon, \delta$, every $t \in \theta$, and a sufficiently large $n$, there is an $\left(n, J_{n}\right)$ code $\left(E_{t},\left\{D_{j}: j=1, \ldots, J_{n}\right\}\right)$ such that $\frac{1}{n} \log J_{n} \geq R-\delta$, and

$$
\begin{gathered}
\max _{t \in \theta} \max _{j \in\left\{1, \ldots, J_{n}\right\}} \sum_{x^{n} \in A^{n}} E_{t}\left(x^{n} \mid j\right) \mathrm{W}_{t}^{n}\left(D_{j}^{c} \mid x^{n}\right) \leq \varepsilon, \\
\max _{t \in \theta} \chi\left(X_{\text {uni }} ; Z_{t}^{n}\right) \leq \varepsilon .
\end{gathered}
$$

Here $Z_{t}^{n}$ are the resulting quantum states at the output of wiretap channels $\mathrm{V}_{t}^{n}$.

A non-negative number $R$ is an achievable secrecy rate for the compound channel with quantum wiretapper $\left(\mathrm{W}_{t}, \mathrm{~V}_{t}\right)_{t \in \theta}$ having no CSI at the encoder, if for every positive $\varepsilon, \delta$ and a sufficiently large $n$, there is an $\left(n, J_{n}\right)$ code $\left(E,\left\{D_{j}: j=1, \ldots, J_{n}\right\}\right)$ such that $\frac{1}{n} \log J_{n} \geq R-\delta$, and

$$
\begin{gathered}
\max _{t \in \theta} \max _{j \in\left\{1, \ldots, J_{n}\right\}} \sum_{x^{n} \in A^{n}} E\left(x^{n} \mid j\right) \mathrm{W}_{t}^{n}\left(D_{j}^{c} \mid x^{n}\right) \leq \varepsilon, \\
\max _{t \in \theta} \chi\left(X_{\text {uni }} ; Z_{t}^{n}\right) \leq \varepsilon .
\end{gathered}
$$

An $\left(n, J_{n}\right)$ code carrying classical information for the compound quantum wiretap channel $\left(W_{t}, V_{t}\right)_{t \in \theta}$ consists of a family of quantum states $\left\{w(j): j=1, \ldots, J_{n}\right\} \subset$ $\mathcal{S}\left(H^{\prime \otimes n}\right)$ and a collection of positive semi-definite operators $\left\{D_{j}: j \in\left\{1, \ldots, J_{n}\right\}\right\}$ on $\mathcal{S}\left(H^{\prime \prime}{ }^{\otimes n}\right)$ which is a partition of the identity, i.e. $\sum_{j=1}^{J_{n}} D_{j}=\mathrm{I}_{H^{\prime \prime} \otimes n}$.

A non-negative number $R$ is an achievable secrecy rate with classical input for the compound quantum wiretap channel $\left(W_{t}, V_{t}\right)_{t \in \theta}$ having CSI at the encoder with average error, if for every positive $\varepsilon, \delta$, every $t \in \theta$, and a sufficiently large $n$, there is an $\left(n, J_{n}\right)$ code carrying classical information $\left(\left\{w_{t}(j): j\right\},\left\{D_{j}: j\right\}\right)$ such that $\frac{1}{n} \log J_{n} \geq R-\delta$, and

$$
\begin{gathered}
\max _{t \in \theta} \frac{1}{J_{n}} \sum_{j=1}^{J_{n}} \operatorname{tr}\left(\left(\mathrm{I}_{H^{\prime \prime} \otimes n}-D_{j}\right) W_{t}^{\otimes n}\left(w_{t}(j)\right)\right) \leq \varepsilon, \\
\max _{t \in \theta} \chi\left(X_{u n i} ; Z_{t}^{n}\right) \leq \varepsilon .
\end{gathered}
$$

A non-negative number $R$ is an achievable secrecy rate with classical input for the compound quantum wiretap channel $\left(W_{t}, V_{t}\right)_{t \in \theta}$ having no CSI at the encoder, if for every positive $\varepsilon$ and $\delta$, and a sufficiently large $n$, there is an $\left(n, J_{n}\right)$ code carrying classical information $(\{w(j)$ : $\left.j\},\left\{D_{j}: j\right\}\right)$ such that $\frac{1}{n} \log J_{n} \geq R-\delta$, and

$$
\begin{gathered}
\max _{t \in \theta} \max _{j \in\left\{1, \ldots, J_{n}\right\}} \operatorname{tr}\left(\left(\mathrm{I}_{H^{\prime \prime} \otimes n}-D_{j}\right) W_{t}^{\otimes n}(w(j))\right) \leq \varepsilon \\
\max _{t \in \theta} \chi\left(X_{\text {uni }} ; Z_{t}^{n}\right) \leq \varepsilon
\end{gathered}
$$

Instead of "achievable secrecy rate with classical input for the compound quantum wiretap channel ", we say $R$ is an achievable secrecy rate for the compound classicalquantum wiretap channel $\left(W_{t}, V_{t}\right)_{t \in \theta}$.

An $\left(n, J_{n}\right)$ code carrying quantum information for the compound quantum channel $\left(N_{t}^{\otimes n}\right)_{t \in \theta}$ consists of a Hilbert spaces $H^{\mathfrak{A}}$ such that $\operatorname{dim} H^{\mathfrak{A}}=J_{n}$, and a general decoding quantum operation $D$, i.e., a completely positive, trace-preserving map $D: \mathcal{S}\left(H^{\mathfrak{Q}^{n}}\right) \rightarrow \mathcal{S}\left(H^{\mathfrak{M}}\right)$, where $H^{\mathfrak{M}}$ is a Hilbert space such that $\operatorname{dim} H^{\mathfrak{M}}=J_{n}$. The code can be used for entanglement generation in the following way. The sender prepares a pure bipartite quantum state $|\psi\rangle^{\mathfrak{A} \mathfrak{P}^{n}}$, defined on $H^{\mathfrak{A}} \otimes H^{\mathfrak{P}^{n}}$, and 
sends the $\mathfrak{P}^{n}$ portion of it through the channel $N_{t}^{\otimes n}$. The receiver performs the general decoding quantum operation on the channel output $D: \mathcal{S}\left(H^{\mathfrak{Q}^{n}}\right) \rightarrow \mathcal{S}\left(H^{\mathfrak{M}}\right)$. The sender and the receiver share the resulting quantum state

$$
\Omega_{t}^{\mathfrak{A M}}:=\left[\mathrm{I}^{\mathfrak{A}} \otimes\left(D \circ N_{t}^{\otimes n}\right)\right]\left(|\psi\rangle\left\langle\left.\psi\right|^{\mathfrak{A P}} \mathfrak{P}^{n}\right) .\right.
$$

A non-negative number $R$ is an achievable entanglement generating rate for the compound quantum channel $\left(N_{t}^{\otimes n}\right)_{t \in \theta}$ if for every positive $\varepsilon, \delta$, and a sufficiently large $n$, there is an $\left(n, J_{n}\right)$ code carrying quantum information $\left(H^{\mathfrak{A}}, D\right)$ such that $\frac{1}{n} \log J_{n} \geq R-\delta$, and

$$
\min _{t \in \theta} F\left(\Omega_{t}^{\mathfrak{A M}},\left|\Phi_{K}\right\rangle\left\langle\left.\Phi_{K}\right|^{\mathfrak{A M}}\right) \geq 1-\varepsilon\right.
$$

where

$$
\left|\Phi_{K}\right\rangle^{\mathfrak{A} \mathfrak{M}}:=\sqrt{\frac{1}{J_{n}}} \sum_{j=1}^{J_{n}}|j\rangle^{\mathfrak{A}}|j\rangle^{\mathfrak{M}}
$$

which is the standard maximally entangled state shared by the sender and the receiver. $\left\{|j\rangle^{\mathfrak{A}}\right\}$ and $\left\{|j\rangle^{\mathfrak{M}}\right\}$ are orthonormal bases for $H^{\mathfrak{A}}$ and $H^{\mathfrak{M}}$, respectively.

The largest achievable secrecy rate is called the secrecy capacity. The largest achievable entanglement generating rate is called the entanglement generating capacity.

\section{CLASSICAL COMPOUND WIRETAP CHANNELS}

In this section, we present some known results for the classical compound wiretap channel which are used for the proof of the results in Section IV.

Let $A, B, C, \theta$, and $\left(\mathrm{W}_{t}, \mathrm{~V}_{t}\right)_{t \in \theta}$ be defined as in Section II. For every $t \in \theta$, we fix a probability distribution $p_{t}$ on $A^{n}$. Let $p_{t}^{\prime}\left(x^{n}\right):= \begin{cases}\frac{p_{t}^{n}\left(x^{n}\right)}{p_{t}^{n}\left(\mathcal{T}_{p_{t}, \delta}^{n}\right)}, & \text { if } x^{n} \in \mathcal{T}_{p_{t}, \delta}^{n} \\ 0, & \text { else }\end{cases}$ and $X^{(t)}:=\left\{X_{j, l}^{(t)}\right\}_{j \in\left\{1, \ldots, J_{n}\right\}, l \in\left\{1, \ldots, L_{n, t}\right\}}$ be a family of random matrices whose entries are selected i.i.d. according to $p_{t}^{\prime}$, where $L_{n, t}$ is a natural number, which will be specified later.

It was shown in [13] that for any positive $\omega$, if we set

$$
J_{n}=\left\lfloor 2^{n\left(\min _{t \in \theta}\left(I\left(p_{t} ; \mathrm{W}_{t}\right)-\frac{1}{n} \log L_{n, t}-\mu\right)\right.}\right\rfloor,
$$

where $\mu$ is a positive constant which does not depend on $j, t$, and can be arbitrarily small when $\omega$ goes to 0 , the following statement is valid. There are such $\left\{D_{j}: j=\right.$ $\left.1, \ldots, J_{n}\right\}$ that for all $t \in \theta$ and for all $L_{n, t} \in \mathbb{N}$

$$
\operatorname{Pr}\left(\max _{j \in\left\{1, \ldots, J_{n}\right\}} \sum_{l=1}^{L_{n, t}} \frac{1}{L_{n, t}} \mathrm{~W}_{t}^{n}\left(D_{j}^{c} \mid X_{j, l}^{(t)}\right)>\sqrt{T} 2^{-n \omega / 2}\right)
$$

$$
\leq \sqrt{T} 2^{-n \omega / 2}
$$

Since only the error of the legitimate receiver is analyzed, for the result (22) just the channels $W_{t}$, but not those of the wiretapper, are regarded. For every $j \in\left\{1, \ldots, J_{n}\right\}$, $l \in\left\{1, \ldots, L_{n, t}\right\}$, and $t \in \theta, \mathrm{W}_{t}^{n}\left(D_{j}^{c} \mid X_{j, l}^{(t)}\right)$ is a random variable taking values in ]0,1[, which depends on $X_{j, l}^{(t)}$, since we defined $X_{j, l}^{(t)}$ as a random variable with value in $A^{n}$.

In view of (22), by choosing $L_{n, t}=\left\lfloor 2^{n\left[I\left(p_{t} ; V_{t}\right)+\tau\right]}\right\rfloor$, for any positive constant $\tau$, the authors of [13] showed that $C_{S, C S I}$, the secrecy capacity of the compound wiretap channel with CSI at the transmitter is given by

$$
C_{S, C S I} \geq \min _{t \in \theta} \max _{\mathcal{U} \rightarrow A \rightarrow(B K)_{t}}\left(I\left(\mathcal{U} ; B_{t}\right)-I\left(\mathcal{U} ; K_{t}\right)\right),
$$

where $B_{t}$ are the resulting random variables at the output of legal receiver channels. $K_{t}$ are the resulting random variables at the output of wiretap channels. The maximum is taken over all random variables that satisfy the Markov chain relationships: $\mathcal{U} \rightarrow A \rightarrow(B Z)_{t}$. Here $A \rightarrow(B Z)_{t}$ means $A \rightarrow B_{t} \times Z_{t}$, where $A \rightarrow B_{t}$ means $A \stackrel{W_{t}}{\longrightarrow} B_{t}$ and $A \rightarrow Z_{t}$ means $A \stackrel{V_{t}}{\longrightarrow} Z_{t}$.

Bjelakovic, Boche and Sommerfeld also proved in [13]

$$
C_{S, C S I} \geq \min _{t \in \theta} \max _{\mathcal{U} \rightarrow A \rightarrow(B K)_{t}}\left(I\left(\mathcal{U} ; B_{t}\right)-I\left(\mathcal{U} ; K_{t}\right)\right) .
$$

Together with this inequality and (23) we have

$$
C_{S, C S I}=\min _{t \in \theta} \max _{\mathcal{U} \rightarrow A \rightarrow(B K)_{t}}\left(I\left(\mathcal{U} ; B_{t}\right)-I\left(\mathcal{U} ; K_{t}\right)\right) .
$$

Analogously, in the case without CSI, the idea is similar to the case with CSI: Fix a probability distribution $p$ on $A^{n}$. Let $p^{\prime}\left(x^{n}\right):= \begin{cases}\frac{p^{n}\left(x^{n}\right)}{p^{n}\left(\mathcal{T}_{p, \delta}^{n}\right)} & \text { if } x^{n} \in \mathcal{T}_{p, \delta}^{n} \\ 0 & \text { else }\end{cases}$

and $X^{n}:=\left\{X_{j, l}\right\}_{j \in\left\{1, \ldots, J_{n}\right\}, l \in\left\{1, \ldots, L_{n}\right\}}$, where $L_{n}$, a natural number, will be specified later, be a family of random matrices whose components are selected i.i.d. according to $p^{\prime}$.

For any $\omega>0$, we define

$$
J_{n}=\left\lfloor 2^{n\left(\min _{t \in \theta}\left(I\left(p ; \mathrm{W}_{t}\right)-\frac{1}{n} \log L_{n}-\mu\right)\right.}\right\rfloor,
$$

where $\mu$ is a positive constant which does not depend on $j$ and $t$, and can be arbitrarily small when $\omega$ goes to 0 . There are $\left\{D_{j}: j=1, \ldots, J_{n}\right\}$ such that for all $t \in \theta$ and for all $L_{n} \in \mathbb{N}$

$$
\begin{aligned}
& \operatorname{Pr}\left(\max _{j \in\left\{1, \ldots, J_{n}\right\}} \sum_{l=1}^{L_{n}} \frac{1}{L_{n}} \mathrm{~W}_{t}^{n}\left(D_{j}^{c} \mid X_{j, l}\right)>\sqrt{T} 2^{-n \omega / 2}\right) \\
& \leq \sqrt{T} 2^{-n \omega / 2} .
\end{aligned}
$$

In view of (25), by choosing $L_{n}=\left\lfloor 2^{n\left[\max _{t} I\left(p_{t} ; V_{t}\right)+\frac{\tau}{4}\right]}\right\rfloor$, where $\tau$ is a positive constant, the authors of [13] showed that $C_{S}$, the secrecy capacity of the compound wiretap channel without CSI at the transmitter, is lower bounded as follows:

$$
C_{S} \geq \max _{\mathcal{U} \rightarrow A \rightarrow(B K)_{t}}\left(\min _{t \in \theta} I\left(\mathcal{U} ; B_{t}\right)-\max _{t \in \theta} I\left(\mathcal{U} ; K_{t}\right)\right) .
$$




\section{COMPOUND CHANNELS WITH QUANTUM WIRETAPPER}

In this section we discuss the classical compound channel with a quantum wiretapper. For the case when the sender has the full knowledge about the CSI, we derive the secrecy capacity. For the case when the sender does not know the CSI, we give a lower bound for the secrecy capacity. In this channel model, the wiretapper uses classical-quantum channels. II

Let $A, B, H, \theta$, and $\left(\mathrm{W}_{t}, \mathrm{~V}_{t}\right)_{t \in \theta}$ be defined as in Section

Theorem 1 The secrecy capacity of the compound channel with quantum wiretapper $\left(\mathrm{W}_{t}, \mathrm{~V}_{t}\right)_{t \in \theta}$ in the case with CSI at the transmitter $C_{S, C S I}$ is given by

$C_{S, C S I}=\min _{t \in \theta} \max _{\mathcal{U} \rightarrow A \rightarrow(B Z)_{t}}\left(I\left(\mathcal{U} ; B_{t}\right)-\limsup _{n \rightarrow \infty} \frac{1}{n} \chi\left(\mathcal{U} ; Z_{t}^{n}\right)\right)$

Respectively, in the case without CSI, the secrecy capacity of the compound channel with quantum wiretapper $\left(\mathrm{W}_{t}, \mathrm{~V}_{t}\right)_{t \in \theta} C_{S}$ is lower bounded as follows

$$
C_{S} \geq \max _{\mathcal{U} \rightarrow A \rightarrow(B Z)_{t}}\left(\min _{t \in \theta} I\left(\mathcal{U} ; B_{t}\right)-\max _{t} \chi\left(\mathcal{U} ; Z_{t}\right)\right),
$$

where $B_{t}$ are the resulting random variables at the output of legal receiver channels, and $Z_{t}$ are the resulting random quantum states at the output of wiretap channels.

Remark 3 We have only the multi-letter formulas 27) and (28), since we do not have a single-letter formula even for a quantum channel which is neither compound nor has wiretappers.

Proof. 1) Lower bound for case with CSI Let

For every $t \in \theta$, fix a probability distribution $p_{t}$ on $A^{n}$.

$$
J_{n}=\left\lfloor 2^{n\left(\min _{t \in \theta}\left(I\left(p_{t} ; \mathrm{W}_{t}\right)-\frac{1}{n} \log L_{n, t}-\mu\right)\right.}\right\rfloor,
$$

where $L_{n, t}$ is a natural number that will be specified below, and $\mu$ is defined as in Section III. Let $p_{t}^{\prime}, X^{(t)}$, and $D_{j}$ be defined as in the classical case. Then (22) still holds, since the sender transmits through a classical channel to the legitimate receiver.

Let

$Q_{t}\left(x^{n}\right):=\Pi_{p_{t} \mathrm{v}_{t}, \alpha \sqrt{a}} \Pi_{\mathrm{V}_{t}, \alpha}\left(x^{n}\right) \cdot \mathrm{V}_{t}^{\otimes n}\left(x^{n}\right) \cdot \Pi_{\mathrm{V}_{t}, \alpha}\left(x^{n}\right) \Pi_{p_{t} \mathrm{~V}_{t}, \alpha \sqrt{a}}$

where $\alpha$ will be defined later.

Lemma 1 (Tender Operator, cf. [41] and [32])

Let $\rho$ be a quantum state and $X$ be a positive operator with $X \leq \mathrm{I}$ and $1-\operatorname{tr}(\rho X) \leq \lambda \leq 1$. Then

$$
\|\rho-\sqrt{X} \rho \sqrt{X}\| \leq \sqrt{2 \lambda} .
$$

Tender Operator was first introduced in [41], where it has been shown that $\|\rho-\sqrt{X} \rho \sqrt{X}\| \leq \sqrt{8 \lambda}$. In [32], the result of [41] has been improved, and (29) has been proved.

In view of the fact that $\Pi_{p_{t} \mathrm{v}_{t}, \alpha \sqrt{a}}$ and $\Pi_{\mathrm{V}_{t}, \alpha}\left(x^{n}\right)$ are both projection matrices, by (1), (7), and Lemma 1 for any $t$ and $x^{n}$, it holds that

$$
\left\|Q_{t}\left(x^{n}\right)-\mathrm{V}_{t}^{\otimes n}\left(x^{n}\right)\right\| \leq \sqrt{\frac{2(a d+d)}{n \alpha^{2}}} .
$$

We set $\Theta_{t}:=\sum_{x^{n} \in \mathcal{T}_{p_{t}, \delta}^{n}} p_{t}^{\prime n}\left(x^{n}\right) Q_{t}\left(x^{n}\right)$. For given $z^{n}$ and $t,\left\langle z^{n}\left|\Theta_{t}\right| z^{n}\right\rangle$ is the expected value of $\left\langle z^{n}\left|Q_{t}\left(x^{n}\right)\right| z^{n}\right\rangle$ under the condition $x^{n} \in \mathcal{T}_{p_{t}, \delta}^{n}$.

Lemma 2 (Covering Lemma, cf. [3]) Let $\mathcal{V}$ be a finite dimensional Hilbert space. Let $\mathcal{E} \subset \mathcal{S}(\mathcal{V})$ be a collection of density operators such that $\sigma \leq \mu \cdot \mathrm{I}_{\mathcal{V}}$ for all $\sigma \in \mathcal{E}$, and let $p$ be a probability distribution on on $\mathcal{E}$. For any positive $\lambda$, we define a sequence of i.i.d. random variables $X_{1}, \ldots, X_{L}$, taking values in $\mathcal{E}$ such that for all $\sigma \in \mathcal{E}$ we have $p(\sigma)=\operatorname{Pr}\left\{X_{i}=\Pi_{\rho, \lambda}^{\prime} \cdot \sigma \cdot \Pi_{\rho, \lambda}^{\prime}\right\}$, where $\rho:=\sum_{\sigma \in \mathcal{E}} p(\sigma) \sigma$, and $\Pi_{\rho, \lambda}^{\prime}$ is the projector onto the subspace spanned by the eigenvectors of $\rho$ whose corresponding eigenvalues are greater than $\frac{\lambda}{\operatorname{dim} \mathcal{V}}$. For any $\epsilon \in] 0,1[$, the following inequality holds

$$
\begin{aligned}
& \operatorname{Pr}\left(\left\|L^{-1} \sum_{i=1}^{L} X_{i}-\Pi_{\rho, \lambda}^{\prime} \cdot \rho \cdot \Pi_{\rho, \lambda}^{\prime}\right\|>\epsilon\right) \\
& \leq 2 \cdot(\operatorname{dim} \mathcal{V}) \exp \left(-L \frac{\epsilon^{2} \lambda}{2 \ln 2(\operatorname{dim} \mathcal{V}) \mu}\right) .
\end{aligned}
$$

Let $\mathcal{V}$ be the range space of $\Pi_{p_{t} \mathrm{v}_{t}, \alpha \sqrt{a}}$. By (2) we have

$$
\operatorname{dim} \mathcal{V} \leq 2^{n S\left(p_{t}\right)+K d \alpha \sqrt{a n}}
$$

Furthermore, for all $x^{n}$ holds

$$
\begin{aligned}
& Q_{t}\left(x^{n}\right) \\
& =\Pi_{p_{t} \mathrm{~V}_{t}, \alpha \sqrt{a}} \Pi_{\mathrm{V}_{t}, \alpha}\left(x^{n}\right) \cdot \mathrm{V}_{t}^{\otimes n}\left(x^{n}\right) \cdot \Pi_{\mathrm{V}_{t}, \alpha}\left(x^{n}\right) \Pi_{p_{t} \mathrm{v}_{t}, \alpha \sqrt{a}} \\
& \leq 2^{-n\left(S\left(\mathrm{v}_{t} \mid p_{t}\right)+K a d \alpha \sqrt{n}\right)} \Pi_{p_{t} \mathrm{v}_{t}, \alpha \sqrt{a}} \Pi_{\mathrm{V}_{t}, \alpha}\left(x^{n}\right) \Pi_{p_{t} \mathrm{v}_{t}, \alpha \sqrt{a}} \\
& \leq 2^{-n \cdot S\left(\mathrm{v}_{t} \mid p_{t}\right)+K a d \alpha \sqrt{n}} \cdot \Pi_{p_{t} \mathrm{v}_{t}, \alpha \sqrt{a}} \\
& \leq 2^{-n \cdot S\left(\mathrm{v}_{t} \mid p_{t}\right)+K a d \alpha \sqrt{n}} \cdot \mathrm{I}_{\mathcal{V}}
\end{aligned}
$$

The first inequality follows from (66). The second inequality holds because $\Pi_{\mathrm{v}_{t}, \alpha}$ and $\Pi_{p_{t} \mathrm{v}_{t}, \alpha \sqrt{a}}$ are projection matrices. The third inequality holds because $\Pi_{p_{t} \mathrm{v}_{t}, \alpha \sqrt{a}}$ is a projection matrix onto $\mathcal{V}$.

Let $\lambda=\epsilon$. By applying Lemma 2, where we set $\mu:=$ $2^{-n \cdot S\left(\mathrm{~V}_{t} \mid p_{t}\right)+K a d \alpha \sqrt{n}}$ in (31) in view of (32), if $n$ is large enough we have

$$
\operatorname{Pr}\left(\left\|\sum_{l=1}^{L_{n, t}} \frac{1}{L_{n, t}} Q_{t}\left(X_{j, l}\right)-\Theta_{t}\right\|>\epsilon\right)
$$




$$
\begin{aligned}
& \leq 2^{n\left(S\left(p_{t}\right)+K d \alpha \sqrt{a n}\right)} \\
& \cdot \exp \left(-L_{n, t} \frac{\epsilon^{2}}{2 \ln 2} \lambda \cdot 2^{n\left(S\left(\mathrm{v}_{t} \mid p_{t}\right)-S\left(p_{t}\right)\right)+K d \alpha \sqrt{n}(\sqrt{a}-1)}\right) \\
& =2^{n\left(S\left(p_{t}\right)+K d \alpha \sqrt{a n}\right)} \\
& \cdot \exp \left(-L_{n, t} \frac{\epsilon^{2}}{2 \ln 2} \lambda \cdot 2^{n\left(-\chi\left(p_{t} ; Z_{t}\right)\right)+K d \alpha \sqrt{n}(\sqrt{a}-1)}\right) \\
& \leq \exp \left(-L_{n, t} \cdot 2^{-n\left(\chi\left(p_{t} ; Z_{t}\right)+\zeta\right)}\right)
\end{aligned}
$$

where $\zeta$ is some suitable positive constant which does not depend on $j, t$, and can be arbitrarily small when $\epsilon$ is close to 0 . The equality in the last line holds since

$$
\begin{aligned}
& S\left(p_{t}\right)-S\left(\mathrm{~V}_{t} \mid p_{t}\right) \\
& =S\left(\sum_{j} p_{t}(j) \sum_{l} \frac{1}{L_{n, t}} \mathrm{~V}_{t}^{\otimes n}\left(X_{j, l}^{(t)}\right)\right) \\
& -\sum_{j} p_{t}(j) S\left(\sum_{l} \frac{1}{L_{n, t}} \mathrm{~V}_{t}^{\otimes n}\left(X_{j, l}^{(t)}\right)\right) \\
& =\chi\left(p_{t} ; Z_{t}\right) .
\end{aligned}
$$

Let $L_{n, t}=\left\lceil 2^{n\left(\chi\left(p_{t} ; Z_{t}\right)+2 \zeta\right)}\right\rceil$, and $n$ be large enough, then by (34) for all $j$ it holds that

$$
\operatorname{Pr}\left(\left\|\sum_{l=1}^{L_{n, t}} \frac{1}{L_{n, t}} Q_{t}\left(X_{j, l}^{(t)}\right)-\Theta_{t}\right\|>\epsilon\right) \leq \exp \left(-2^{n \zeta}\right)
$$

and

$$
\begin{aligned}
& \operatorname{Pr}\left(\left\|\sum_{l=1}^{L_{n, t}} \frac{1}{L_{n, t}} Q_{t}\left(X_{j, l}^{(t)}\right)-\Theta_{t}\right\| \leq \epsilon \forall t \forall j\right) \\
& =1-\operatorname{Pr}\left(\bigcup_{t}\left\{\bigcup_{j}\left\{\sum_{l=1}^{L_{n, t}} \frac{1}{L_{n, t}} Q_{t}\left(X_{j, l}^{(t)}\right)-\Theta_{t} \|>\epsilon\right\}\right)\right. \\
& \geq 1-T J_{n} \exp \left(-2^{n \zeta}\right) \\
& \geq 1-T 2^{n\left(\min _{t \in \theta}\left(I\left(p_{t} ; \mathrm{W}_{t}\right)-\frac{1}{n} \log L_{n, t}\right)\right.} \exp \left(-2^{n \zeta}\right) \\
& \geq 1-2^{-n v},
\end{aligned}
$$

where $v$ is some suitable positive constant which does not depend on $j$ and $t$.

Remark 4 Since exp $\left(-2^{n \zeta}\right)$ converges to zero double exponentially quickly, the inequality (36) remains true even if $T$ depends on $n$ and is exponentially large over $n$, i.e., we can still achieve an exponentially small error.

From (22) and (36) it follows: For any $\epsilon>0$, if $n$ is large enough then the event

$$
\begin{array}{r}
\left(\bigcap_{t}\left\{\max _{j \in\left\{1, \ldots, J_{n}\right\}} \sum_{l=1}^{L_{n, t}} \frac{1}{L_{n, t}} \mathrm{~W}_{t}^{n}\left(D_{j}^{c}(\mathcal{X}) \mid X_{j, l}^{(t)}\right) \leq \epsilon\right\}\right) \\
\cap\left(\left\{\left\|\sum_{l=1}^{L_{n, t}} \frac{1}{L_{n, t}} Q_{t}\left(X_{j, l}^{(t)}\right)-\Theta_{t}\right\| \leq \epsilon \forall t \forall j\right\}\right)
\end{array}
$$

has a positive probability. This means that we can find a realization $x_{j, l}^{(t)}$ of $X_{j, l}^{(t)}$ with a positive probability such that for all $t \in \theta$ and $j \in\left\{1, \ldots, J_{n}\right\}$, we have

$$
\sum_{l=1}^{L_{n, t}} \frac{1}{L_{n, t}} \mathrm{~W}_{t}^{n}\left(D_{j}^{c} \mid x_{j, l}^{(t)}\right) \leq \epsilon
$$

and

$$
\left\|\sum_{l=1}^{L_{n, t}} \frac{1}{L_{n, t}} Q_{t}\left(x_{j, l}^{(t)}\right)-\Theta_{t}\right\| \leq \epsilon .
$$

For an arbitrary $\gamma>0$ let

$R:=\min _{t \in \theta} \max _{\mathcal{U} \rightarrow A \rightarrow(B Z)_{t}}\left(I\left(\mathcal{U} ; B_{t}\right)-\limsup _{n \rightarrow \infty} \frac{1}{n} \chi\left(\mathcal{U} ; Z_{t}^{n}\right)\right)-\gamma$.

Choose $\mu<\frac{1}{2} \gamma$, then for every $t \in \theta$, there is an $\left(n, J_{n}\right)$ code $\quad\left(\left(x_{j, l}^{(t)}\right)_{j=1, \ldots, J_{n}, l=1, \ldots, L_{n, t}},\left\{D_{j}: j=1, \ldots, J_{n}\right\}\right)$ such that

$$
\frac{1}{n} \log J_{n} \geq R
$$

$$
\lim _{n \rightarrow \infty} \max _{t \in \theta} \max _{j \in\left\{1, \ldots, J_{n}\right\}} \sum_{l=1}^{L_{n, t}} \frac{1}{L_{n, t}} \mathrm{~W}_{t}^{n}\left(D_{j}^{c} \mid x_{j, l}^{(t)}\right)=0 .
$$

Choose a suitable $\alpha$ in (30) such that for all $j$, it holds $\left\|\mathrm{V}_{t}^{\otimes n}\left(x_{j, l}^{(t)}\right)-Q_{t}\left(x_{j, l}^{(t)}\right)\right\|<\epsilon$. For any given $j^{\prime} \in$ $\left\{1, \ldots, J_{n}\right\}$, (30) and (38) yield

$$
\begin{aligned}
& \left\|\sum_{l=1}^{L_{n, t}} \frac{1}{L_{n, t}} \mathrm{~V}_{t}^{\otimes n}\left(x_{j^{\prime}, l}^{(t)}\right)-\Theta_{t}\right\| \\
& \leq\left\|\sum_{l=1}^{L_{n, t}} \frac{1}{L_{n, t}} \mathrm{~V}_{t}^{\otimes n}\left(x_{j^{\prime}, l}^{(t)}\right)-\sum_{l=1}^{L_{n, t}} \frac{1}{L_{n, t}} Q_{t}\left(x_{j^{\prime}, l}^{(t)}\right)\right\| \\
& +\left\|\sum_{l=1}^{L_{n, t}} \frac{1}{L_{n, t}} Q_{t}\left(x_{j^{\prime}, l}^{(t)}\right)-\Theta_{t}\right\| \\
& \leq \sum_{l=1}^{L_{n, t}} \frac{1}{L_{n, t}}\left\|\mathrm{~V}_{t}^{\otimes n}\left(x_{j^{\prime}, l}^{(t)}\right)-Q_{t}\left(x_{j^{\prime}, l}^{(t)}\right)\right\| \\
& +\left\|\sum_{l=1}^{L_{n, t}^{(t)}} \frac{1}{L_{n, t}} Q_{t}\left(x_{j^{\prime}, l}^{(t)}\right)-\Theta_{t}\right\| \\
& \leq 2 \epsilon,
\end{aligned}
$$

and $\left\|\sum_{j=1}^{J_{n}} \frac{1}{J_{n}} \sum_{l=1}^{L_{n, t}} \frac{1}{L_{n, t}} \mathrm{~V}_{t}^{\otimes n}\left(x_{j, l}^{(t)}\right)-\Theta_{t}\right\| \leq \epsilon$. 
Lemma 3 (Fannes-Audenaert Ineq., cf. [22], [4]) Let $\Phi$ and $\Psi$ be two quantum states in a d-dimensional complex Hilbert space and $\|\Phi-\Psi\| \leq \mu<\frac{1}{e}$, then

$|S(\Phi)-S(\Psi)| \leq \mu \log (d-1)-\mu \log \mu-(1-\mu) \log (1-\mu)$.

The Fannes Inequality was first introduced in 22], where it has been shown that $|S(\mathfrak{X})-S(\mathfrak{Y})| \leq \mu \log d-$ $\mu \log \mu$. In [4] the result of [22] has been improved, and (42) has been proved.

By Lemma 3 and the inequality (41), for a uniformly distributed distributed random variable $X_{u n i}$ with value in $\left\{1, \ldots, J_{n}\right\}$, we have

$$
\begin{aligned}
& \chi\left(X_{u n i} ; Z_{t}^{n}\right) \\
& =S\left(\sum_{j=1}^{J_{n}} \frac{1}{J_{n}} \sum_{l=1}^{L_{n, t}} \frac{1}{L_{n, t}} \mathrm{~V}_{t}^{\otimes n}\left(x_{j, l}^{(t)}\right)\right) \\
& -\sum_{j=1}^{J_{n}} \frac{1}{J_{n}} S\left(\sum_{l=1}^{L_{n, t}} \frac{1}{L_{n, t}} \mathrm{~V}_{t}^{\otimes n}\left(x_{j, l}^{(t)}\right)\right) \\
& \leq\left|S\left(\sum_{j=1}^{J_{n}} \frac{1}{J_{n}} \sum_{l=1}^{L_{n, t}} \frac{1}{L_{n, t}} \mathrm{~V}_{t}^{\otimes n}\left(x_{j, l}^{(t)}\right)\right)-S\left(\Theta_{t}\right)\right| \\
& +\left|S\left(\Theta_{t}\right)-\sum_{j=1}^{J_{n}} \frac{1}{J_{n}} S\left(\sum_{l=1}^{L_{n, t}} \frac{1}{L_{n, t}} \mathrm{~V}_{t}^{\otimes n}\left(x_{j, l}^{(t)}\right)\right)\right| \\
& \leq \epsilon \log (d-1)-\epsilon \log \epsilon-(1-\epsilon) \log (1-\epsilon) \\
& +\left|\sum_{j=1}^{J_{n}} \frac{1}{J_{n}}\left[S\left(\Theta_{t}\right)-S\left(\sum_{l=1}^{L_{n, t}} \frac{1}{L_{n, t}} \mathrm{~V}_{t}^{\otimes n}\left(x_{j, l}^{(t)}\right)\right)\right]\right| \\
& \leq 3 \epsilon \log (d-1)-\epsilon \log \epsilon-(1-\epsilon) \log (1-\epsilon)-2 \epsilon \log 2 \epsilon .
\end{aligned}
$$

By (43), for any positive $\lambda$ if $n$ is sufficiently large, we have

$$
\max _{t \in \theta} \chi\left(X_{u n i} ; Z_{t}^{n}\right) \leq \lambda .
$$

For every $t \in \theta$ we define an $\left(n, J_{n}\right)$ code $\left(E_{t},\left\{D_{j}: j=1, \ldots, J_{n}\right\}\right)$, where $E_{t}$ is built such that $\operatorname{Pr}\left(E_{t}(j)=x_{j, l}^{(t)}\right)=\frac{1}{L_{n, t}}$ for $l \in\left\{1, \ldots, L_{n, t}\right\}$. Combining (40) and (44) we obtain

$C_{S, C S I} \geq \min _{t \in \theta} \max _{\mathcal{U} \rightarrow A \rightarrow(B Z)_{t}}\left(I\left(\mathcal{U} ; B_{t}\right)-\limsup _{n \rightarrow \infty} \frac{1}{n} \chi\left(\mathcal{U} ; Z_{t}^{n}\right)\right)$

Thus, we have shown the " $\geq$ " part of (27).

2) Upper bound for case with CSI

Let $\left(\mathcal{C}_{n}\right)$ be a sequence of $\left(n, J_{n}\right)$ codes such that

$$
\max _{t \in \theta} \max _{j \in\left\{1, \ldots, J_{n}\right\}} \sum_{x^{n} \in A^{n}} E\left(x^{n} \mid j\right) \mathrm{W}_{t}^{n}\left(D_{j}^{c} \mid x^{n}\right)=: \epsilon_{1, n},
$$

$$
\max _{t \in \theta} \chi\left(J ; Z_{t}^{n}\right)=: \epsilon_{2, n},
$$

where $\lim _{n \rightarrow \infty} \epsilon_{1, n}=0$ and $\lim _{n \rightarrow \infty} \epsilon_{2, n}=0 . J$ denotes the random variable which is uniformly distributed on the message set $\left\{1, \ldots, J_{n}\right\}$.

We denote the security capacity of the wiretap channel $\left(\mathrm{W}_{t}, \mathrm{~V}_{t}\right)$ in the sense of [40] by $C\left(\mathrm{~W}_{t}, \mathrm{~V}_{t}\right)$. Choose $t^{\prime} \in \theta$ such that $C\left(\mathrm{~W}_{t^{\prime}}, \mathrm{V}_{t^{\prime}}\right)=\min _{t \in \theta} C\left(\mathrm{~W}_{t}, \mathrm{~V}_{t}\right)$.

We denote a new random variable by $\hat{X}$ with values in $\left\{1, \ldots, J_{n}\right\}$ determined by the Markov chain $X_{u n i} \rightarrow$ $A \rightarrow B_{t^{\prime}} \rightarrow \hat{X}$, where the first transition is governed by the sender's encoding strategy, the second by $W_{t^{\prime}}$, and the last by the legal receiver's decoding strategy. Then we have from the data processing inequality

$$
\begin{aligned}
& \log J_{n}=H\left(X_{u n i}\right) \\
& =I\left(X_{u n i}, \hat{X}\right)+H\left(X_{u n i} \mid \hat{X}\right) \\
& \leq I\left(X_{u n i}, B_{t^{\prime}}^{n}\right)+H\left(X_{u n i} \mid \hat{X}\right) .
\end{aligned}
$$

Using Fano's inequality we have

$$
H\left(X_{\text {uni }} \mid \hat{X}\right) \leq 1+\epsilon_{1, n} \log J_{n} .
$$

Thus $\log J_{n} \leq I\left(X_{u n i}, B_{t^{\prime}}^{n}\right)+1+\epsilon_{1, n} \log J_{n}$. Applying the standard technique for single letter formula in classical information theory we have

$$
\log J_{n} \leq n I\left(X_{\text {uni }}, B_{t^{\prime}}\right)+1+\epsilon_{1, n} \log J_{n} .
$$

Thus for any $\epsilon>0$, if $n$ is sufficiently large $\frac{1}{n} \log J_{n}$ can not be greater than

$$
\begin{aligned}
& I\left(X_{\text {uni }} ; B_{t^{\prime}}\right)+\frac{1}{n}+\frac{1}{n} \epsilon_{1, n} \log J_{n} \\
& \leq\left[I\left(X_{u n i} ; B_{t^{\prime}}\right)-\frac{1}{n} \chi\left(X_{u n i} ; Z_{t^{\prime}}^{n}\right)\right]+\frac{\epsilon_{1, n}}{n}+\frac{1}{n} \log J_{n}+\frac{\epsilon_{2, n}}{n} \\
& \leq\left[I\left(X_{u n i} ; B_{t^{\prime}}\right)-\frac{1}{n} \chi\left(X_{u n i} ; Z_{t^{\prime}}^{n}\right)\right]+\epsilon .
\end{aligned}
$$

We can not exceed the secrecy capacity of the worst wiretap channel, since we have to guarantee that the legal receiver can decode the message in the worst case (cf. (13) and Section (I). Thus, we have

$C_{S, C S I} \leq \min _{t \in \theta} \max _{\mathcal{U} \rightarrow A \rightarrow(B Z)_{t}}\left(I\left(\mathcal{U} ; B_{t}\right)-\limsup _{n \rightarrow \infty} \frac{1}{n} \chi\left(\mathcal{U} ; Z_{t}^{n}\right)\right)$.

Combining (50) and (45) we obtain (27).

3) Lower bound for case without CSI

Fix a probability distribution $p$ on $A^{n}$. Let

$$
J_{n}=\left\lfloor 2^{\min _{t \in \theta}\left(n I\left(p ; \mathrm{W}_{t}\right)-\log L_{n}\right)-n \mu}\right\rfloor,
$$

where $L_{n}$ is a natural defined as in Section III. Let $p^{\prime}$, $X^{n}$, and $D_{j}$ (25) still holds.

For a positive $\alpha$, we define

$Q_{t}\left(x^{n}\right):=\Pi_{p \mathrm{v}_{t}, \alpha \sqrt{a}} \Pi_{\mathrm{v}_{t}, \alpha}\left(x^{n}\right) \cdot \mathrm{v}_{t}^{\otimes n}\left(x^{n}\right) \cdot \Pi_{\mathrm{V}_{t}, \alpha}\left(x^{n}\right) \Pi_{p \mathrm{v}_{t}, \alpha \sqrt{a}}$ 
and $\Theta_{t}:=\sum_{x^{n} \in \mathcal{T}_{p, \delta}^{n}} p^{\prime n}\left(x^{n}\right) Q_{t}\left(x^{n}\right)$.

For any positive $\delta$ let $L_{n}=\left\lceil 2^{n \max _{t}\left(\chi\left(p ; Z_{t}\right)+\delta\right)}\right\rceil$ and $n$ be large enough, in the same way as our proof of (36) for the case with CSI at the encoder, there is a positive constant $v$ so that

$$
\operatorname{Pr}\left(\left\|\sum_{l=1}^{L_{n}} \frac{1}{L_{n}} Q_{t}\left(X_{j, l}^{(t)}\right)-\Theta_{t}\right\| \leq \epsilon \forall t \forall j\right) \geq 1-2^{-n v} .
$$

For any positive $\epsilon$ we choose a suitable $\alpha$, by (25) and (51) there is a realization $x_{j, l}$ of $X_{j, l}$ with a positive probability such that: For all $t \in \theta$ and all $j \in\left\{1, \ldots J_{n}\right\}$, we have

$$
\begin{gathered}
\sum_{l=1}^{L_{n}} \frac{1}{L_{n}} \mathrm{~W}_{t}^{n}\left(D_{j}^{c} \mid x_{j, l}\right) \leq \epsilon, \\
\left\|\sum_{l=1}^{L_{n}} \frac{1}{L_{n}} Q_{t}\left(x_{j, l}\right)-\Theta_{t}\right\| \leq \epsilon .
\end{gathered}
$$

For any $\gamma>0$ let

$$
R:=\max _{\mathcal{U} \rightarrow A \rightarrow(B Z)_{t}}\left(\min _{t \in \theta} I\left(\mathcal{U} ; B_{t}\right)-\max _{t} \chi\left(\mathcal{U} ; Z_{t}\right)\right)-\gamma .
$$

Then there is an $\left(n, J_{n}\right)$ code $\left(E,\left\{D_{j}: j=1, \ldots, J_{n}\right\}\right)$, where $E$ is so built that $\operatorname{Pr}\left(E(j)=x_{j, l}\right)=\frac{1}{L_{n, t}}$ for $l \in$ $\left\{1, \ldots, L_{n, t}\right\}$, such that $\liminf _{n \rightarrow \infty} \frac{1}{n} \log J_{n} \geq R$, and

$$
\left.\lim _{n \rightarrow \infty} \max _{t \in \theta} \max _{j \in\left\{1, \ldots, J_{n}\right\}} \sum_{l=1}^{L_{n}} \frac{1}{L_{n}} \mathrm{~W}_{t}^{n}\left(D_{j}^{c} \mid x_{j, l}\right)\right)=0 .
$$

In the same way as our proof of (44) for the case with CSI at the encoder,

$$
\max _{t \in \theta} \chi\left(X_{u n i} ; Z_{t}^{n}\right) \leq \epsilon,
$$

for any uniformly distributed distributed random variable $X_{\text {uni }}$ with value in $\left\{1, \ldots, J_{n}\right\}$.

Combining (52) and (53) we obtain

$$
C_{S} \geq \max _{\mathcal{U} \rightarrow A \rightarrow(B Z)_{t}}\left(\min _{t \in \theta} I\left(\mathcal{U} ; B_{t}\right)-\max _{t \in \theta} \chi\left(\mathcal{U} ; Z_{t}\right)\right) .
$$

\section{COMPOUND CLASSICAL-QUANTUM WIRETAP CHANNEL}

In this section, we derive the secrecy capacity of the compound classical-quantum wiretap channel with CSI. In this model, both the receiver and the wiretapper use classical quantum channels and the set of the channel states may be finite or infinite.

Let $A, H, H^{\prime}, H^{\prime \prime}, \theta$, and $\left(W_{t}, V_{t}\right)_{t \in \theta}$ be defined as in Section [1]
Theorem 2 The secrecy capacity of the compound classical-quantum wiretap channel in the case with CSI is given by

$$
C_{C S I}=\lim _{n \rightarrow \infty} \min _{t \in \theta} \max _{P_{\text {inp }}, w_{t}} \frac{1}{n}\left(\chi\left(P_{\text {inp }} ; B_{t}^{n}\right)-\chi\left(P_{\text {inp }} ; Z_{t}^{n}\right)\right)
$$

where $B_{t}$ are the resulting random quantum states at the output of legal receiver channels and $Z_{t}$ are the resulting random quantum states at the output of wiretap channels. The maximum is taken over all probability distributions $P_{\text {inp }}$ on the input quantum states $w_{t}$.

Assume that the sender's encoding is restricted to transmitting an indexed finite set of orthogonal quantum states $\left\{\rho_{x}: x \in A\right\} \subset \mathcal{S}\left(H^{\prime \otimes n}\right)$, then the secrecy capacity of the compound classical-quantum wiretap channel in the case with no CSI at the encoder is given by

$$
\begin{aligned}
& C_{S}=\lim _{n \rightarrow \infty} \max _{\mathcal{U} \rightarrow A \rightarrow(B Z)_{t}} \frac{1}{n}\left(\min _{t \in \theta} \chi\left(\mathcal{U} ; B_{t}^{n}\right)\right. \\
& \left.-\max _{t \in \theta} \chi\left(\mathcal{U} ; Z_{t}^{n}\right)\right) .
\end{aligned}
$$

Proof. At first we are going to prove (54). Our idea is to send the information in two parts. First, we send the channel state information with finite blocks of finite bits with a code $C_{1}$ to the receiver, and then, depending on $t$, we send the message with a code $C_{2}^{(t)}$ in the second part.

\section{1) Sending channel state information with finite bits}

We do not require that the first part should be secure against the wiretapper, since we assume that the wiretapper already has the full knowledge of the CSI.

By ignoring the security against the wiretapper, we consider only the compound channel $\left(W_{t}\right)_{t \in \theta}$. Let $W=$ $\left(W_{t}\right)_{t}$ be an arbitrary compound-classical quantum channel. Then, by [9], for each $\lambda \in(0,1)$, the $\lambda$ capacity $C(W, \lambda)$ equals

$$
C(W, \lambda)=\max _{P_{i n p} \in P(A)} \min _{t} \chi\left(P_{i n p} ; W_{t}\right) .
$$

If $\max _{P_{\text {inp }}} \min _{t} \chi\left(P_{\text {inp }} ; W_{t}\right)>0$ holds, then the sender can build a code $C_{1}$ such that the CSI can be sent to the legal receiver with a block with length $l \leq$ $\frac{\log T}{\min _{t} \max _{P_{i n p}} \chi\left(P_{i n p}, W_{t}\right)}-\epsilon$. If $\max _{P_{i n p}} \min _{t} \chi\left(P_{i n p} ; W_{t}\right)=$ 0 holds, we can not build a code $C_{1}$ such that the CSI can be sent to the legal receiver. But, this does not cause any problem, since if $\max _{P_{i n p}} \min _{t} \chi\left(P_{i n p} ; W_{t}\right)=0$, the right-hand side of (54) is zero.

1.2) Message transformation when both the sender and the legal receiver know CSI

If both the sender and the legal receiver have the full knowledge of $t$, then we only have to look at the single wiretap channel $\left(W_{t}, V_{t}\right)$.

In [19] and [20] it was shown that if $n$ is sufficiently large, there exists an $\left(n, J_{n}\right)$ code for the quantum wiretap channel $(W, V)$ with

$$
\log J_{n}=\max _{P_{\text {inp }}, w}\left(\chi\left(P_{\text {inp }} ; B^{n}\right)-\chi\left(P_{\text {inp }} ; Z^{n}\right)\right)-\epsilon,
$$


for any positive $\epsilon$ and positive $\delta$, where $B$ is the resulting random variable at the output of legal receiver's channel and $Z$ the output of the wiretap channel.

When the sender and the legal receiver both know $t$, they can build an $\left(n, J_{n, t}\right)$ code $C_{2}^{(t)}$ where

$$
\log J_{n, t}=\max _{P_{i n p}, w_{t}}\left(\chi\left(P_{i n p} ; B_{t}^{n}\right)-\chi\left(P_{i n p} ; Z_{t}^{n}\right)\right)-\epsilon .
$$

Thus,

$C_{C S I} \geq \lim _{n \rightarrow \infty} \min _{t \in \theta} \max _{P_{\text {inp }}, w_{t}} \frac{1}{n}\left(\chi\left(P_{\text {inp }} ; B_{t}^{n}\right)-\chi\left(P_{\text {inp }} ; Z_{t}^{n}\right)\right)$.

Remark 5 For the construction of the second part of our code, we use random coding and request that the randomization can be sent (cf. [19]]). However, it was shown in [13] that the randomization could not always be sent if we require that we use one unique code which is secure against the wiretapper and suitable for every channel state, i.e., it does not depend on $t$. This is not a counterexample to our results above, neither to the construction of $C_{1}$ nor to the construction of $C_{2}^{(t)}$, because of the following facts.

The first part of our code does not need to be secure. For our second part, the legal transmitters can use the following strategy: At first they build a code $C_{1}=\left(E,\left\{D_{t}: t=1, \ldots,|\theta|\right\}\right)$ and a code $C_{2}^{(t)}=\left(E^{(t)},\left\{D_{j}^{(t)}: j=1, \ldots, J_{n}\right\}\right)$ for every $t \in \theta$. If the sender wants to send the CSI $t^{\prime} \in \theta$ and the message $j$, he encodes $t^{\prime}$ with $E$ and $j$ with $E^{\left(t^{\prime}\right)}$, then he sends both parts together through the channel. After receiving both parts, the legal receiver decodes the first part with $\left\{D_{t}: t\right\}$, and chooses the right decoders $\left\{D_{j}^{\left(t^{\prime}\right)}: j\right\} \in\left\{\left\{D_{j}^{(t)}: j\right\}: t \in \theta\right\}$ to decode the second part. With this strategy, we can avoid using one unique code which is suitable for every channel state.

\section{3) Upper bound for the case CSI at the encoder}

For any $\epsilon>0$, we choose $t^{\prime} \in \theta$ such that $C\left(W_{t^{\prime}}, V_{t^{\prime}}\right) \leq$ $\inf _{t \in \theta} C\left(W_{t}, V_{t}\right)+\epsilon$.

From [19] and [20] we know that the secrecy capacity of the quantum wiretap channel $\left(W_{t^{\prime}}, V_{t^{\prime}}\right)$ can not be greater than

$$
\lim _{n \rightarrow \infty} \max _{P_{\text {inp }}, w_{t^{\prime}}} \frac{1}{n}\left(\chi\left(P_{\text {inp }} ; B_{t^{\prime}}^{n}\right)-\chi\left(P_{\text {inp }} ; Z_{t^{\prime}}^{n}\right)\right) .
$$

Since we can not exceed the capacity of the worst wiretap channel, we have

$$
C_{C S I} \leq \lim _{n \rightarrow \infty} \min _{t \in \theta} \max _{P_{\text {inp }}, w_{t}} \frac{1}{n}\left(\chi\left(P_{\text {inp }} ; B_{t}^{n}\right)-\chi\left(P_{\text {inp }} ; Z_{t}^{n}\right)\right) .
$$

This together with (59) completes the proof of (54).
Remark 6 In [39] it was shown that if for a given $t$ and any $n \in \mathbb{N}$,

$$
\chi\left(P_{\text {inp }} ; B_{t}^{n}\right) \geq \chi\left(P_{\text {inp }} ; Z_{t}^{n}\right)
$$

holds for all $P_{\text {inp }} \in P(A)$ and $\left\{w_{t}(j): j=1, \ldots, J_{n}\right\} \subset$ $S\left(H^{\otimes n}\right)$, then

$$
\begin{aligned}
& \lim _{n \rightarrow \infty} \max _{P_{\text {inp }}, w_{t}} \frac{1}{n}\left(\chi\left(P_{\text {inp }} ; B_{t}^{n}\right)-\chi\left(P_{\text {inp }} ; Z_{t}^{n}\right)\right) \\
& =\max _{P_{\text {inp }}, w_{t}}\left(\chi\left(P_{\text {inp }} ; B_{t}\right)-\chi\left(P_{\text {inp }} ; Z_{t}\right)\right) .
\end{aligned}
$$

Thus if for every $t \in \theta$ and $n \in \mathbb{N}$,

$$
I\left(P_{\text {inp }}, B_{t}^{n}\right) \geq I\left(P_{\text {inp }} ; Z_{t}^{n}\right)
$$

holds for all $P_{\text {inp }} \in P(A)$ and $\left\{w_{t}(j): j=1, \ldots, J_{n}\right\} \subset$ $S\left(H^{\otimes n}\right)$, we have

$$
C_{C S I}=\min _{t \in \theta} \max _{P_{i n p}, w_{t}}\left(\chi\left(P_{i n p} ; B_{t}\right)-\chi\left(P_{\text {inp }} ; Z_{t}\right)\right) .
$$

Now we are going to prove (55).

2.1) Lower bound for case without CSI

Fix a probability distribution $p$ on $A^{n}$. Let

$$
\begin{gathered}
J_{n}=\left\lfloor 2^{\min _{t \in \theta} \chi\left(p ; B_{t}^{n}\right)-\max _{t \in \theta} \chi\left(p ; Z_{t}^{n}\right)-2 n \mu}\right\rfloor, \\
L_{n}=\left\lceil 2^{\max _{t} \chi\left(p ; Z_{t}^{n}\right)+n \mu}\right\rceil,
\end{gathered}
$$

and let $p^{\prime}$ and $X^{n}=\left\{X_{j, l}: j, l\right\}$ be defined as in the classical case (cf. Section III). Since $J_{n} \cdot L_{n} \leq$ $2^{\min _{t} \chi\left(p ; B_{t}^{n}\right)-n \mu}$, in [18] it was shown that if $n$ is sufficiently large, there exist a collection of quantum states $\left\{\rho_{x^{n}}: x^{n} \in A^{n}\right\} \subset \mathcal{S}\left(H^{\prime \otimes n}\right)$, a collection of positivesemidefinite operators $\left\{D_{t, x^{n}}: t \in \theta, x^{n} \in A^{n}\right\}$, and a positive constant $\beta$, such that for any $(t, j, l) \in \theta \times$ $\left\{1, \ldots, J_{n}\right\} \times\left\{1, \ldots, L_{n}\right\}$ it holds

$$
\operatorname{Pr}\left[\operatorname{tr}\left(W_{t}^{n}\left(\rho_{X_{j, l}}^{n}\right) D_{t, X_{j, l}}\right) \geq 1-2^{-n \beta}\right]>1-2^{-n \beta},
$$

and for any realization $\left\{x_{j, l}: j, l\right\}$ of $\left\{X_{j, l}: j, l\right\}$ it holds that

$$
\sum_{t \in \theta} \sum_{j=1}^{J_{n}} \sum_{l=1}^{L_{n}} D_{t, x_{j, l}} \leq \mathrm{I} .
$$

We define

$Q_{t}\left(\rho_{x^{n}}\right):=\Pi_{p V_{t}, \alpha \sqrt{a}} \Pi_{V_{t}, \alpha}\left(x^{n}\right) \cdot V_{t}^{\otimes n}\left(\rho_{x^{n}}\right) \cdot \Pi_{V_{t}, \alpha}\left(x^{n}\right) \Pi_{p V_{t}, \alpha \sqrt{a}}$, and $\Theta_{t}:=\sum_{x^{n} \in \mathcal{T}_{p, \delta}^{n}} p^{\prime n}\left(x^{n}\right) Q_{t}\left(\rho_{x^{n}}\right)$.

Choosing $n$ sufficiently large, in the same way as our proof of (36) for the classical compound channel with 
quantum wiretapper, there is a positive constant $v$ such that

$$
\operatorname{Pr}\left(\left\|\sum_{l=1}^{L_{n}} \frac{1}{L_{n}} Q_{t}\left(\rho_{X_{j, l}^{(t)}}\right)-\Theta_{t}\right\| \leq \epsilon \forall t \forall j\right) \geq 1-2^{-n v} .
$$

We choose a suitable $\alpha$. If $n$ is sufficiently large, we can find a realization $x_{j, l}$ of $X_{j, l}$ with a positive probability such that for all $j \in\left\{1, \ldots J_{n}\right\}$, we have

$$
\min _{t \in \theta} \operatorname{tr}\left(W_{t}^{n}\left(\rho_{x_{j, l}}^{n}\right) D_{t, x_{j, l}}\right) \geq 1-2^{-n \beta}
$$

and

$$
\max _{t \in \theta}\left\|\sum_{l=1}^{L_{n}} \frac{1}{L_{n}} Q_{t}\left(\rho_{x_{j, l}}\right)-\Theta_{t}\right\| \leq \epsilon .
$$

We define $D_{j}:=\sum_{t \in \theta} \sum_{l=1}^{L_{n}} D_{t, x_{j, l}}$, then $\sum_{j=1}^{J_{n}} D_{j}=$ $\sum_{t \in \theta} \sum_{j=1}^{J_{n}} \sum_{l=1}^{L_{n}} D_{t, x_{j, l}} \leq \mathrm{I}$. Furthermore, for all $t^{\prime} \in \theta$ and $l^{\prime} \in\left\{1, \ldots, L_{n}\right\}$ we have

$$
\begin{aligned}
& \operatorname{tr}\left(W_{t^{\prime}}^{n}\left(\rho_{x_{j, l^{\prime}}}^{n}\right) D_{j}\right) \\
& =\sum_{t \in \theta} \sum_{l=1}^{L_{n}} \operatorname{tr}\left(W_{t^{\prime}}^{n}\left(\rho_{x_{j, l^{\prime}}}^{n}\right) D_{t, x_{j, l}}\right) \\
& \geq \operatorname{tr}\left(W_{t^{\prime}}^{n}\left(\rho_{x_{j, l^{\prime}}}^{\otimes n}\right) D_{t^{\prime}, x_{j, l^{\prime}}}\right) \\
& \geq 1-2^{-n \beta},
\end{aligned}
$$

the inequality in the third line holds because for two positive semi-definite matrices $M_{1}$ and $M_{2}$, we always have $\operatorname{tr}\left(M_{1} M_{2}\right)=\operatorname{tr}\left(\sqrt{M_{1}} M_{2} \sqrt{M_{1}}\right) \geq 0$.

For any $\gamma>0$ let

$$
R:=\max _{\mathcal{U} \rightarrow A \rightarrow(B Z)_{t}} \frac{1}{n}\left[\min _{t \in \theta} \chi\left(p ; B_{t}^{n}\right)-\max _{t \in \theta} \chi\left(p ; Z_{t}^{n}\right)\right]-\gamma .
$$

Then for any positive $\lambda$, there is an $\left(n, J_{n}, \lambda\right)$ code $\left(\left\{w(j):=\sum_{l=1}^{L_{n}} \frac{1}{L_{n}} \rho_{x_{j, l}}^{n}: j=1, \ldots, J_{n},\right\},\left\{D_{j}: j=\right.\right.$ $\left.\left.1, \ldots, J_{n}\right\}\right)$, such that $\liminf _{n \rightarrow \infty} \frac{1}{n} \log J_{n} \geq R$,

$$
\max _{t \in \theta} \max _{j \in\left\{1, \ldots, J_{n}\right\}} \operatorname{tr}\left(\left(\mathrm{I}_{H^{\prime \prime} \otimes n}-D_{j}\right) W_{t}^{\otimes n}(w(j))\right) \leq \lambda,
$$

and in the same way as our proof of (44) for the classical compound channel with quantum wiretapper,

$$
\max _{t \in \theta} \chi\left(X_{u n i} ; Z_{t}^{n}\right) \leq \lambda,
$$

for any uniformly distributed random variable $X_{u n i}$ with value in $\left\{1, \ldots, J_{n}\right\}$.

Combining (63) and (64) we obtain

$C_{S} \geq \lim _{n \rightarrow \infty} \max _{\mathcal{U} \rightarrow(B Z)_{t}} \frac{1}{n}\left(\min _{t \in \theta} \chi\left(\mathcal{U} ; B_{t}^{n}\right)-\max _{t \in \theta} \chi\left(\mathcal{U} ; Z_{t}^{n}\right)\right)$.

\section{2) Upper bound for case without CSI}

Let $\left(\mathcal{C}_{n}\right)=\left(\left\{\rho_{j}^{(n)}: j\right\},\left\{D_{j}^{(n)}: j\right\}\right)$ be a sequence of $\left(n, J_{n}, \lambda_{n}\right)$ code such that

$$
\begin{gathered}
\max _{t \in \theta} \max _{j \in\left\{1, \ldots, J_{n}\right\}} \operatorname{tr}\left(\left(\mathrm{I}-D_{j}^{(n)}\right) W_{t}^{\otimes n}\left(\rho_{j}^{(n)}\right)\right) \leq \lambda_{n}, \\
\max _{t \in \theta} \chi\left(X_{\text {uni }} ; Z_{t}^{n}\right)=: \epsilon_{2, n},
\end{gathered}
$$

where $\lim _{n \rightarrow \infty} \lambda_{n}=0$ and $\lim _{n \rightarrow \infty} \epsilon_{2, n}=0$. $X_{\text {uni }}$ denotes the random variable which is uniformly distributed on the message set $\left\{1, \ldots, J_{n}\right\}$.

We denote the classical capacity of the quantum channel $W_{t}$ in the sense of [40] by $C\left(W_{t}\right)$. Choose $t^{\prime} \in \theta$ such that $C\left(W_{t^{\prime}}\right)=\min _{t \in \theta} C\left(W_{t}\right)$.

It is known (cf. Section IV 2) Upper bound for case with CSI and [31] $)$ that can not exceed $\chi\left(X_{u n i} ; B_{t^{\prime}}^{n}\right)+\xi$ for any constant $\xi>0$. Since the secrecy capacity of a compound wiretap channel can not exceed the capacity of the worst channel without wiretapper, for any $\epsilon>0$ choose $\xi=\frac{1}{2} \epsilon$, if $n$ is large enough, the secrecy rate of $\left(\mathcal{C}_{n}\right)$ can not be greater than

$$
\begin{aligned}
& \frac{1}{n} \chi\left(X_{u n i} ; B_{t^{\prime}}^{n}\right)+\xi \\
& =\min _{t \in \theta} \frac{1}{n} \chi\left(X_{u n i} ; B_{t}^{n}\right)+\xi \\
& \leq \min _{t \in \theta} \frac{1}{n} \chi\left(X_{u n i} ; B_{t}^{n}\right)-\max _{t \in \theta} \frac{1}{n} \chi\left(X_{u n i} ; Z_{t}^{n}\right)+\xi+\frac{1}{n} \epsilon_{2, n} \\
& \leq \frac{1}{n}\left(\min _{t \in \theta} \chi\left(X_{u n i} ; B_{t}^{n}\right)-\max _{t \in \theta} \chi\left(X_{u n i} ; Z_{t}^{n}\right)\right)+\epsilon .
\end{aligned}
$$

Thus

$C_{S} \leq \lim _{n \rightarrow \infty} \max _{\mathcal{U} \rightarrow A \rightarrow(B Z)_{t}} \frac{1}{n}\left(\min _{t \in \theta} \chi\left(\mathcal{U} ; B_{t}^{n}\right)-\max _{t \in \theta} \chi\left(\mathcal{U} ; Z_{t}^{n}\right)\right)$.

Combining (69) and (65) we obtain (55).

So far, we assumed that $|\theta|$, the number of the channels, is finite, therefore we can send the CSI with finite bits to the receiver in the case where the sender has CSI. Now we look at the case where $|\theta|$ can be arbitrary. We of course are not allowed to send the CSI with finite bits if $|\theta|=\infty$, but in this case, we may use a "finite approximation" to obtain the following corollary.

Corollary 1 For an arbitrary set $\theta$ we have

$C_{S, C S I}=\lim _{n \rightarrow \infty} \inf _{t \in \theta} \max _{P_{\text {inp }}, w_{t}} \frac{1}{n}\left(\chi\left(P_{i n p} ; B_{t}^{n}\right)-\chi\left(P_{i n p} ; Z_{t}^{n}\right)\right)$.

Proof. Let $W: \mathcal{S}\left(H^{\prime}\right) \rightarrow \mathcal{S}\left(H^{\prime \prime}\right)$ be a linear map, then let

$$
\|W\|_{\diamond}:=\sup _{n \in \mathbb{N}} \max _{a \in S\left(\mathbb{C}^{n} \otimes H^{\prime}\right),\|a\|_{1}=1}\left\|\left(\mathrm{I}_{n} \otimes W\right)(a)\right\|_{1} .
$$


It is known [33] that this norm is multiplicative, i.e. $\left\|W \otimes W^{\prime}\right\|_{\diamond}=\|W\|_{\diamond} \cdot\left\|W^{\prime}\right\|_{\diamond}$.

A $\tau$-net in the space of the completely positive trace-preserving maps $\mathcal{S}\left(H^{\prime}\right) \rightarrow \mathcal{S}\left(H^{\prime \prime}\right)$ is a finite set $\left(W^{(k)}\right)_{k=1}^{K}$ of completely positive trace-preserving maps $\mathcal{S}\left(H^{\prime}\right) \rightarrow \mathcal{S}\left(H^{\prime \prime}\right)$ with the property that for each completely positive trace-preserving map $W: \mathcal{S}\left(H^{\prime}\right) \rightarrow$ $\mathcal{S}\left(H^{\prime \prime}\right)$, there is at least one $k \in\{1, \ldots, K\}$ with $\| W-$ $W^{(k)} \|_{\diamond}<\tau$.

Lemma 4 ( $\tau$-net [30]) Let $H^{\prime}$ and $H^{\prime \prime}$ be finitedimensional complex Hilbert spaces. For any $\tau \in(0,1]$, there is a $\tau$-net of quantum-channels $\left(W^{(k)}\right)_{k=1}^{K}$ in the space of the completely positive trace preserving maps $\mathcal{S}\left(H^{\prime}\right) \rightarrow \mathcal{S}\left(H^{\prime \prime}\right)$ with $K \leq\left(\frac{3}{\tau}\right)^{2 d^{\prime 4}}$, where $d^{\prime}=\operatorname{dim} H^{\prime}$.

If $|\theta|$ is arbitrary, then for any $\xi>0$ let $\tau=\frac{\xi}{-\log \xi}$. By Lemma 4 there exists a finite set $\theta^{\prime}$ with $\left|\theta^{\prime}\right| \leq\left(\frac{3}{\tau}\right)^{2 d^{\prime 4}}$ and $\tau$-nets $\left(W_{t^{\prime}}\right)_{t^{\prime} \in \theta^{\prime}},\left(V_{t^{\prime}}\right)_{t^{\prime} \in \theta^{\prime}}$ such that for every $t \in \theta$ we can find a $t^{\prime} \in \theta^{\prime}$ with $\left\|W_{t}-W_{t^{\prime}}\right\|_{\diamond} \leq \tau$ and $\left\|V_{t}-V_{t^{\prime}}\right\|_{\diamond} \leq \tau$. For every $t^{\prime} \in \theta^{\prime}$, the legal transmitters build a code $C_{2}^{\left(t^{\prime}\right)}=\left\{w_{t^{\prime}},\left\{D_{t^{\prime}, j}: j\right\}\right\}$. Since by [19], the error probability of the code $C_{2}^{\left(t^{\prime}\right)}$ decreases exponentially with its length, there is an $N=O(-\log \xi)$ such that for all $t^{\prime \prime} \in \theta^{\prime}$ it holds

$$
\begin{gathered}
\frac{1}{J_{N}} \sum_{j=1}^{J_{N}} \operatorname{tr}\left(W_{t^{\prime \prime}}^{\otimes N}\left(w_{t^{\prime \prime}}(j)\right) D_{t^{\prime \prime}, j}\right) \geq 1-\lambda-\xi, \\
\chi\left(X_{\text {uni }} ; Z_{t^{\prime}}^{N}\right) \leq \xi .
\end{gathered}
$$

Then, if the sender obtains the channel state information " $t$ ", he chooses a " $t$ " $\in \theta^{\prime}$ such that $\left\|W_{t}-W_{t^{\prime}}\right\|_{\diamond} \leq$ $\tau$ and $\left\|V_{t}-V_{t^{\prime}}\right\|_{\diamond} \leq \tau$. He can send " $t$ " to the legal receiver in the first part with finite bits, and then they build a code $C_{2}^{\left(t^{\prime}\right)}$ that fulfills (72) and (73) to transmit the message.

For every $t^{\prime}$ and $j$ let $\left|\psi_{t^{\prime}}(j)\right\rangle\left\langle\psi_{t^{\prime}}(j)\right| \in \mathcal{S}\left(H^{\prime \otimes N} \otimes\right.$ $\left.H^{\prime \otimes N}\right)$ be an arbitrary purification of the quantum state $w_{t^{\prime}}(j)$, then $\operatorname{tr}\left[\left(W_{t}^{\otimes N}-W_{t^{\prime}}^{\otimes N}\right)\left(w_{t^{\prime}}(j)\right)\right]=$ $\operatorname{tr}\left(\operatorname{tr}_{H^{\prime} \otimes N}\left[\mathrm{I}_{H^{\prime}}^{\otimes N} \otimes\left(W_{t}^{\otimes N}-W_{t^{\prime}}^{\otimes N}\right)\left(\left|\psi_{t^{\prime}}(j)\right\rangle\left\langle\psi_{t^{\prime}}(j)\right|\right)\right]\right)$.

We have

$$
\begin{aligned}
& \operatorname{tr}\left|\left(W_{t}^{\otimes N}-W_{t^{\prime}}^{\otimes N}\right)\left(w_{t^{\prime}}(j)\right)\right| \\
& =\operatorname{tr}\left(\operatorname{tr}_{H^{\prime} \otimes N}\left|\mathrm{I}_{H^{\prime}}^{\otimes N} \otimes\left(W_{t}^{\otimes N}-W_{t^{\prime}}^{\otimes N}\right)\left(\left|\psi_{t^{\prime}}(j)\right\rangle\left\langle\psi_{t^{\prime}}(j)\right|\right)\right|\right) \\
& =\operatorname{tr}\left|\mathrm{I}_{H^{\prime}}^{\otimes N} \otimes\left(W_{t}^{\otimes n}-W_{t^{\prime}}^{\otimes N}\right)\left(\left|\psi_{t^{\prime}}(j)\right\rangle\left\langle\psi_{t^{\prime}}(j)\right|\right)\right| \\
& =\left\|\mathrm{I}_{H^{\prime}}^{\otimes N} \otimes\left(W_{t}^{\otimes N}-W_{t^{\prime}}^{\otimes N}\right)\left(\left|\psi_{t^{\prime}}(j)\right\rangle\left\langle\psi_{t^{\prime}}(j)\right|\right)\right\|_{1} \\
& \leq\left\|W_{t}^{\otimes N}-W_{t^{\prime}}^{\otimes N}\right\|_{\diamond} \cdot\left\|\left(\left|\psi_{t^{\prime}}(j)\right\rangle\left\langle\psi_{t^{\prime}}(j)\right|\right)\right\|_{1} \\
& \leq N \tau .
\end{aligned}
$$

The second equality follows from the definition of trace. The second inequality follows by the definition of $\|\cdot\|_{\diamond}$. The third inequality follows from the facts that $\left\|\left(\left|\psi_{t^{\prime}}(j)\right\rangle\left\langle\psi_{t^{\prime}}(j)\right|\right)\right\|_{1}=1$ and $\left\|W_{t}^{\otimes N}-W_{t^{\prime}}^{\otimes N}\right\|_{\diamond}=$ $\left\|\left(W_{t}-W_{t^{\prime}}\right)^{\otimes N}\right\|_{\diamond}=N \cdot\left\|W_{t}-W_{t^{\prime}}\right\|_{\diamond}$, since $\|\cdot\|_{\diamond}$ is multiplicative.

It follows that

$$
\begin{aligned}
& \mid \frac{1}{J_{N}} \sum_{j=1}^{J_{N}} \operatorname{tr}\left(W_{t}^{\otimes N}\left(w_{t^{\prime}}(j)\right) D_{t^{\prime}, j}\right) \\
& -\frac{1}{J_{N}} \sum_{j=1}^{J_{N}} \operatorname{tr}\left(W_{t^{\prime}}^{\otimes N}\left(w_{t^{\prime}}(j)\right) D_{t^{\prime}, j}\right) \mid \\
& \leq \frac{1}{J_{N}} \sum_{j=1}^{J_{N}}\left|\operatorname{tr}\left[\left(W_{t}^{\otimes N}-W_{t^{\prime}}^{\otimes N}\right)\left(w_{t^{\prime}}(j)\right) D_{t^{\prime}, j}\right]\right| \\
& \leq \frac{1}{J_{N}} \sum_{j=1}^{J_{N}} \operatorname{tr}\left|\left(W_{t}^{\otimes N}-W_{t^{\prime}}^{\otimes N}\right)\left(w_{t^{\prime}}(j)\right) D_{t^{\prime}, j}\right| \\
& \leq \frac{1}{J_{N}} \sum_{j=1}^{J_{N}} \operatorname{tr}\left|\left(W_{t}^{\otimes N}-W_{t^{\prime}}^{\otimes N}\right)\left(w_{t^{\prime}}(j)\right)\right| \\
& \leq \frac{1}{J_{N}} J_{N} N \tau \\
& =N \tau
\end{aligned}
$$

$N \tau$ can be arbitrarily small when $\xi$ is close to zero, since $N=O(-\log \xi)$.

Let $X_{\text {uni }}$ be a random variable uniformly distributed on $\left\{1, \ldots, J_{N}\right\}$, and $\left\{\rho(j): j=1, \ldots, J_{n}\right\}$ be a set of quantum states labeled by elements of $\left\{1, \ldots, J_{n}\right\}$. We have

$$
\begin{aligned}
& \left|\chi\left(X_{\text {uni } i} ; V_{t}\right)-\chi\left(X_{\text {uni }} ; V_{t^{\prime}}\right)\right| \\
& \leq\left|S\left(\sum_{j=1}^{J_{N}} \frac{1}{J_{N}} V_{t}(\rho(j))\right)-S\left(\sum_{j=1}^{J_{N}} \frac{1}{J_{N}} V_{t^{\prime}}(\rho(j))\right)\right| \\
& +\left|\sum_{j=1}^{J_{N}} \frac{1}{J_{N}} S\left(V_{t}(\rho(j))\right)-\sum_{j=1}^{J_{N}} \frac{1}{J_{N}} S\left(V_{t^{\prime}}(\rho(j))\right)\right| \\
& \leq \tau \log (d-1)-\tau \log \tau-(1-\tau) \log (1-\tau),
\end{aligned}
$$

where $d=\operatorname{dim} H$. The inequality in the last line holds by Lemma 3 and because $\left\|V_{t}(\rho)-V_{t^{\prime}}(\rho)\right\| \leq \tau$ for all $\rho \in \mathcal{S}(H)$ when $\left\|V_{t}-V_{t^{\prime}}\right\|_{\diamond} \leq \tau$.

By (74) and (75) we have

$$
\begin{gathered}
\sup _{t \in \theta} \frac{1}{J_{N}} \sum_{j=1}^{J_{N}} \operatorname{tr}\left(W_{t}^{\otimes N}\left(w_{t^{\prime}}(j)\right) D_{t^{\prime}, j}\right) \geq 1-\lambda-\xi-N \tau, \\
\chi\left(X_{\text {uni }} ; Z_{t}^{N}\right) \leq \xi+\tau \log (d-1)-\tau \log \tau-(1-\tau) \log (1-\tau) .
\end{gathered}
$$

Since $\xi+N \tau$ and $\tau \log (d-1)$ can be arbitrarily small, when $\xi$ is close to zero, we have

$$
\sup _{t \in \theta} \frac{1}{J_{N}} \sum_{j=1}^{J_{N}} \operatorname{tr}\left(W_{t}^{\otimes N}\left(w_{t^{\prime}}(j)\right) D_{t^{\prime}, j}\right) \geq 1-\lambda,
$$




$$
\sup _{t \in \theta} \chi\left(X_{u n i} ; Z_{t}^{N}\right) \leq \epsilon .
$$

The bits that the sender uses to transform the CSI are large but constant, so it is still negligible compared to the second part. We obtain

$$
C_{C S I} \geq \lim _{n \rightarrow \infty} \inf _{t \in \theta} \max _{P_{\text {inp }}, w_{t}} \frac{1}{n}\left(\chi\left(P_{\text {inp }} ; B_{t}^{n}\right)-\chi\left(P_{\text {inp }} ; Z_{t}^{n}\right)\right) .
$$

The proof of the converse is similar to those given in the proof of Theorem 2, where we consider a worst $t^{\prime}$.

Remark 7 In (54) and Corollary 11 we have only required that the legal receiver can decode the correct message with a high probability if $n$ is sufficiently large. We have not specified how fast the error probability tends to zero when the code length goes to infinity. If we analyze the relation between the error probability $\varepsilon$ and the code length, then we have the following facts.

In the case of finite $\theta$, let $\varepsilon_{1}$ denote the error probability of the first part of the code (i.e. the legal receiver does not decode the correct CSI), and let $\varepsilon_{2}$ denote the error probability of the second part of the code (i.e. the legal receiver decodes the correct CSI, but does not decode the message). Since the length of the first part of the code is $l \cdot \log c \cdot c^{\prime}=O\left(\log \varepsilon_{1}\right)$, we have $\varepsilon_{1}^{-1}$ is $O(\exp (l \cdot \log c \cdot$ $\left.\left.c^{\prime}\right)\right)=O(\exp (n))$, where $n$ stands for the length of the first part of the code. For the second part of the code, $\varepsilon_{2}$ decreased exponentially with the length of the second part, as proven in [19]. Thus, the error probability $\varepsilon=$ $\max \left\{\varepsilon_{1}, \varepsilon_{2}\right\}$ decreases exponentially with the code length in the case of finite $\theta$.

If $\theta$ is infinite, let $\varepsilon_{1}$ denote the error probability of the first part of the code probability. Here we have to build two $\tau$-nets for a suitable $\tau$, each contains $O\left(\left(\frac{-\log \varepsilon_{1}}{\varepsilon}\right)^{-2 d^{\prime 4}}\right)$ channels. If we want to send the CSI of these $\tau$-nets, the length of first part $l$ will be $O\left(-2 d^{\prime 4} \cdot \log \left(\varepsilon_{1} \log \varepsilon_{1}\right)\right)$, which means here $\varepsilon_{1}^{-1}$ will be $O\left(\exp \left(\frac{n}{4 d^{\prime 4}}\right)\right)=O(\exp (n))$. Thus we can still achieve that the error probability decreases exponentially with the code length in case of infinite $\theta$.

\section{ENTANGLEMENT GENERATION OVER COMPOUND QUANTUM CHANNELS}

The entanglement generating capacity of a given quantum channel describes the maximal amount of entanglement that we can generate or transmit over the channel. A code for the secure message transmission over a classical-quantum wiretap channel can be used to build a code for the entanglement transmission over a quantum channel (cf. 20]). Our technique for entanglement generation over compound quantum channels is similar to the proof of entanglement generating capacity over quantum channels in [20]. The difference between our technique and the proofs in [20] is that we have to consider the channel uncertainty (c.f. the discussion in Section VII).
Let $\mathfrak{P}, \mathfrak{Q}, H^{\mathfrak{P}}, H^{\mathfrak{Q}}, \theta$, and $\left(N_{t}^{\otimes n}\right)_{t \in \theta}$ be defined as in Section (i.e., we assume that $\theta$ is finite).

We denote $\operatorname{dim} H^{\mathfrak{P}}$ by $a$, and denote $\mathcal{X}:=\{1, \ldots, a\}$. Consider the eigen-decomposition of $\rho^{\mathfrak{P}}$ into the orthonormal pure quantum state ensemble $\left\{p(x),\left|\phi_{x}\right\rangle^{\mathfrak{P}}\right.$ : $x \in \mathcal{X}\}$,

$$
\sum_{x \in \mathcal{X}} p(x)\left|\phi_{x}\right\rangle\left\langle\left.\phi_{x}\right|^{\mathfrak{P}}=\rho^{\mathfrak{P}}\right.
$$

The distribution $p$ defines a random variable $X$.

Theorem 3 The entanglement generating capacity of $\left(N_{t}\right)_{t \in \theta}$ is bounded as follows

$$
A \geq \max _{p}\left(\min _{t \in \theta} \chi\left(p ; Q_{t}\right)-\max _{t \in \theta} \chi\left(p ; E_{t}\right)\right),
$$

where $Q_{t}$ stands for the quantum outputs that the receiver observes at the channel state $t$, and $E_{t}$ the quantum outputs at the environment.

(Theorem 3 is weaker than the result in [12], the reason is that we use for our proof a different quantum channel representation. For details and the result in [12] cf. Section VII.)

Proof. Let $\rho^{\mathfrak{P}} \rightarrow U_{N_{t}} \rho^{\mathfrak{P}} U_{N_{t}}^{*}$ be a unitary transformation which represents $N_{t}$ (cf. Section VII), where $U_{N_{t}}$ is a linear operator $\mathcal{S}\left(H^{\mathfrak{P}}\right) \rightarrow \mathcal{S}\left(H^{\mathfrak{Q} \mathfrak{E}}\right)$, and $\mathfrak{E}$ is the quantum system of the environment. Fix a $\rho^{\mathfrak{P}}$ with eigen-decomposition $\sum_{x \in \mathcal{X}} p(x)\left|\phi_{x}\right\rangle^{\mathfrak{P}}\left\langle\left.\phi_{x}\right|^{\mathfrak{P}}\right.$. If the channel state is $t$, the local output density matrix seen by the receiver is

$$
\operatorname{tr}_{\mathfrak{E}}\left(\sum_{x} p(x) U_{N_{t}}\left|\phi_{x}\right\rangle\left\langle\left.\phi_{x}\right|^{\mathfrak{P}} U_{N_{t}}^{*}\right),\right.
$$

and the local output density matrix seen by the environment (which we interpret as the wiretapper) is

$$
\operatorname{tr}_{\mathfrak{Q}}\left(\sum_{x} p(x) U_{N_{t}}\left|\phi_{x}\right\rangle\left\langle\left.\phi_{x}\right|^{\mathfrak{P}} U_{N_{t}}^{*}\right) .\right.
$$

Therefore $\left(N_{t}\right)_{t \in \theta}$ defines a compound classical-quantum wiretap channel $\left(W_{N_{t}}, V_{N_{t}}\right)_{t \in \theta}$, where $W_{N_{t}}: H^{\mathfrak{P}} \rightarrow H^{\mathfrak{Q}}$, $\sum_{x \in \mathcal{X}} p(x)\left|\phi_{x}\right\rangle\left\langle\left.\phi_{x}\right|^{\mathfrak{P}} \rightarrow \operatorname{tr}_{\mathfrak{E}}\left(\sum_{x} p(x) U_{N_{t}}\left|\phi_{x}\right\rangle\left\langle\left.\phi_{x}\right|^{\mathfrak{P}} U_{N_{t}}^{*}\right)\right.\right.$, and $V_{N_{t}}: H^{\mathfrak{P}} \rightarrow H^{\mathfrak{Q}}, \quad \sum_{x \in \mathcal{X}} p(x)\left|\phi_{x}\right\rangle\left\langle\left.\phi_{x}\right|^{\mathfrak{P}} \rightarrow\right.$ $\operatorname{tr}_{\mathfrak{E}}\left(\sum_{x} p(x) U_{N_{t}}\left|\phi_{x}\right\rangle\left\langle\phi_{x}\right|{ }^{\mathfrak{P}} U_{N_{t}}^{*}\right)$.

1) Building the encoder and the first part of the decoding operator

Let

$$
J_{n}=\left\lceil 2^{n\left[\min _{t} \chi\left(X ; Q_{t}\right)-\max _{t} \chi\left(X ; E_{t}\right)-2 \delta\right]}\right\rceil,
$$

and

$$
L_{n}=\left\lceil 2^{n\left(\max _{t} \chi\left(X ; E_{t}\right)+\delta\right)}\right\rceil .
$$


For the compound classical-quantum wiretap channel $\left(W_{N_{t}}, V_{N_{t}}\right)_{t \in \theta}$, since

$$
\begin{aligned}
& \left|\left\{(j, l): j=1, \ldots, J_{n}, l=1, \ldots, L_{n}\right\}\right| \\
& =J_{n} \cdot L_{n} \leq 2^{n \min _{t}\left[\chi\left(X ; Q_{t}\right)-\delta\right]},
\end{aligned}
$$

if $n$ is large enough, by Theorem 2 and [18], the following holds. There is a collection of quantum states $\left\{\rho_{x_{j, l}}^{\mathfrak{P}^{n}}\right.$ : $\left.j=1, \ldots, J_{n}, l=1, \ldots, L_{n}\right\} \subset \mathcal{S}\left(H^{\mathfrak{P}^{n}}\right)$, a collection of positive-semidefinite operators $\left\{D_{t, j, l}:=D_{t, x_{j, l}}: t \in\right.$ $\left.\theta, j=1, \ldots, J_{n}, l=1, \ldots, L_{n}\right\}$, a positive constant $\beta$, and a quantum state $\xi_{t}^{\mathfrak{E}^{n}}$ on $H^{\mathfrak{E}^{n}}$, such that

$$
\operatorname{tr}\left(\left(D_{t, x_{j, l}}^{\mathfrak{Q}^{n}} \otimes \mathrm{I}^{\mathfrak{E}^{n}}\right) U_{N_{t}} \rho_{x_{j, l}}^{\mathfrak{P}^{n}} U_{N_{t}}^{*}\right) \geq 1-2^{-n \beta},
$$

and

$$
\left\|\omega_{j, t}^{\mathfrak{E}^{n}}-\xi_{t}^{\mathfrak{E}^{n}}\right\|_{1}<\epsilon
$$

where $\omega_{j, t}^{\mathfrak{E}^{n}}:=\frac{1}{L_{n, t}} \sum_{l=1}^{L_{n, t}} \operatorname{tr}_{\mathfrak{Q}^{n}}\left(U_{N_{t}} \rho_{x_{j, l}}^{\mathfrak{P}^{n}} U_{N_{t}}^{*}\right)$.

Now the quantum state $\rho_{x_{j, l}}^{\mathfrak{P}^{n}}$ may be pure or mixed. Assume $\rho_{x_{j, l}}^{\mathfrak{P}^{n}}$ is a mixed quantum state $\sum_{i=1}^{n} p^{\prime}{ }_{j, l}(i)\left|\varkappa_{x_{j, l}}^{(i)}\right\rangle\left\langle\left.\varkappa_{x_{j, l}}^{(i)}\right|^{\mathfrak{P}^{n}}\right.$, then

$$
\begin{aligned}
& \sum_{i=1}^{n} p^{\prime}{ }_{j, l}(i) \operatorname{tr}\left(\left(D_{t, x_{j, l}}^{\mathfrak{Q}^{n}} \otimes \mathrm{I}^{\mathfrak{E}^{n}}\right) U_{N_{t}}\left|\varkappa_{x_{j, l}}^{(i)}\right\rangle\left\langle\varkappa_{x_{j, l}}^{(i)}\right| \mathfrak{P}^{n} U_{N_{t}}^{*}\right) \\
& \operatorname{tr}\left(\left(D_{t, x_{j, l}}^{\mathfrak{Q}^{n}} \otimes \mathrm{I}^{\mathfrak{E}^{n}}\right) U_{N_{t}}\left(\sum_{i=1}^{n} p^{\prime}{ }_{j, l}(i)\left|\varkappa_{x_{j, l}}^{(i)}\right\rangle\left\langle\varkappa_{x_{j, l}}^{(i)}\right| \mathfrak{P}^{n}\right) U_{N_{t}}^{*}\right) \\
& \geq 1-2^{-n \beta} .
\end{aligned}
$$

Thus, for all $i$ such that $p^{\prime}{ }_{j, l}(i) \geq \frac{2^{-n \beta}}{1-2^{-n \beta}}$ it must hold $\operatorname{tr}\left(\left(D_{t, x_{j, l}}^{\mathfrak{Q}^{n}} \otimes \mathrm{I}^{\mathfrak{E}^{n}}\right) U_{N_{t}}\left|\varkappa_{x_{j, l}}^{(i)}\right\rangle\left\langle\left.\varkappa_{x_{j, l}}^{(i)}\right|^{\mathfrak{P}^{n}} U_{N_{t}}^{*}\right) \geq 1-2^{-n \beta}\right.$.

If $n$ is large enough, then there is at least one $i_{l, j} \in$ $\{1, \ldots, n\}$ such that $p^{\prime}{ }_{j, l}\left(i_{l, j}\right) \geq \frac{2^{-n \beta}}{1-2^{-n \beta}}$. By Theorem 2, there is a $\xi_{t}^{\mathfrak{E}^{n}}$ on $H^{\mathfrak{E}^{n}}$, such that

$\left\|\frac{1}{L_{n, t}} \sum_{l=1}^{L_{n, t}} \operatorname{tr}_{\mathfrak{Q}^{n}}\left(U_{N_{t}}\left|\varkappa_{x_{j, l}}^{\left(i_{l, j}\right)}\right\rangle\left\langle\left.\varkappa_{x_{j, l}}^{\left(i_{l, j}\right)}\right|^{\mathfrak{P}^{n}} U_{N_{t}}^{*}\right)-\xi_{t}^{\mathfrak{E}^{n}} \|_{1}<\epsilon\right.\right.$.

Thus,

$$
\left(\left\{\left|\varkappa_{x_{j, l}}^{\left(i_{l, j}\right)}\right\rangle\left\langle\left.\varkappa_{x_{j, l}}^{\left(i_{l, j}\right)}\right|^{\mathfrak{P}^{n}}: j, l\right\},\left\{D_{t, x_{j, l}}^{\mathfrak{Q}^{n}}: j, l, t\right\}\right)\right.
$$

is a code with the same security rate as

$$
\left(\left\{\rho_{x_{j, l}}^{\mathfrak{P}^{n}}: j, l\right\},\left\{D_{t, x_{j, l}}^{\mathfrak{Q}^{n}}: j, l, t\right\}\right) .
$$

Hence we may assume that $\rho_{x_{j, l}}^{\mathfrak{P}^{n}}$ is a pure quantum state.

Assume $\rho_{x_{j, l}}^{\mathfrak{P}^{n}}=\left|\varkappa_{j, l}\right\rangle\left\langle\left.\varkappa_{j, l}\right|^{\mathfrak{P}^{n}}\right.$. Let $H^{\mathfrak{M}}$ be a $J_{n^{-}}$ dimensional Hilbert space with an orthonormal basis
$\left\{|j\rangle^{\mathfrak{M}}: j=1, \ldots, J_{n}\right\}, H^{\mathfrak{L}}$ be a $L_{n}$-dimensional Hilbert space with an orthonormal basis $\left\{|l\rangle^{\mathfrak{L}}: l=1, \ldots, L_{n, t}\right\}$, and $H^{\theta}$ be a $|\theta|$-dimensional Hilbert space with an orthonormal basis $\left\{|t\rangle^{\theta}: t \in \theta\right\}$. Let $|0\rangle^{\mathfrak{M}}|0\rangle^{\mathfrak{L}}|0\rangle^{\theta}$ be the ancillas on $H^{\mathfrak{M}}, H^{\mathfrak{L}}$, and $H^{\theta}$, respectively, that the receiver adds. We can (cf. 31]) define a unitary matrix $V^{\mathfrak{Q}^{n} \mathfrak{M} \mathfrak{L} \theta}$ on $H^{\mathfrak{Q}^{n} \mathfrak{M} \mathfrak{L} \theta}$ such that for any given quantum state $\rho^{\mathfrak{Q}^{n}} \in \mathcal{S}\left(H^{\mathfrak{Q}^{n}}\right)$ we have

$$
\begin{aligned}
& V^{\mathfrak{Q}^{n} \mathfrak{M} \mathfrak{L} \theta}\left(\rho^{\mathfrak{Q}^{n}} \otimes|0\rangle\left\langle\left. 0\right|^{\mathfrak{M}} \otimes \mid 0\right\rangle\left\langle\left. 0\right|^{\mathfrak{L}} \otimes \mid 0\right\rangle\left\langle\left. 0\right|^{\theta}\right)\left(V^{\mathfrak{Q}^{n} \mathfrak{M} \mathfrak{L} \theta}\right)^{*}\right. \\
& =\sum_{t} \sum_{j} \sum_{l}\left(D_{t, x_{j, l}}^{\mathfrak{Q}^{n}} \rho^{\mathfrak{Q}^{n}}\right) \otimes|j\rangle\left\langle\left. j\right|^{\mathfrak{M}} \mid l\right\rangle\left\langle\left. l\right|^{\mathfrak{L}} \mid t\right\rangle\left\langle\left. t\right|^{\theta} .\right.
\end{aligned}
$$

We denote

$$
\begin{aligned}
& \psi_{j, l, t}^{\mathfrak{Q}^{n} \mathfrak{E}^{n} \mathfrak{M} \mathfrak{L} \theta} \\
& :=\left(\mathrm{I}^{\mathfrak{E}^{n}} \otimes V^{\mathfrak{Q}^{n} \mathfrak{M} \mathfrak{L} \theta}\right)\left(U_{N} \otimes \mathrm{I}^{\mathfrak{M} \mathfrak{L} \theta}\right)\left[| \varkappa _ { j , l } \rangle \left\langle\left.\varkappa_{j, l}\right|^{\mathfrak{P}^{n}}\right.\right. \\
& \otimes|0\rangle\left\langle\left. 0\right|^{\mathfrak{M}} \otimes \mid 0\right\rangle\left\langle\left. 0\right|^{\mathfrak{L}} \otimes \mid 0\right\rangle\left\langle\left. 0\right|^{\theta}\right]\left(U_{N} \otimes \mathrm{I}^{\mathfrak{M} \mathfrak{L} \theta}\right)^{*} \\
& \left(\mathrm{I}^{\mathfrak{E}^{n}} \otimes V^{\mathfrak{Q}^{n} \mathfrak{M} \mathfrak{L} \theta}\right)^{*},
\end{aligned}
$$

in view of (78), we have

$$
\begin{aligned}
& F\left(\operatorname{tr}_{\mathfrak{Q}^{n} \mathfrak{E} n}\left(\psi_{j, l, t}^{\mathfrak{Q}^{n} \mathfrak{E}^{n} \mathfrak{M} \mathfrak{L} \theta}\right),|j\rangle\left\langle\left. j\right|^{\mathfrak{M}} \otimes \mid l\right\rangle\left\langle\left. l\right|^{\mathfrak{L}} \otimes \mid t\right\rangle\left\langle\left. t\right|^{\theta}\right)\right. \\
& \geq 1-\epsilon .
\end{aligned}
$$

By Uhlmann's theorem (cf. e.g. [40]) we can find a $\left|\zeta_{j, l, t}\right\rangle^{\mathfrak{Q}^{n} \mathfrak{E}^{n}}$ on $H^{\mathfrak{Q}^{n} \mathfrak{E}^{n}}$, such that

$$
\begin{aligned}
& \left\langle0 | ^ { \theta } \left\langle0 | ^ { \mathfrak { L } } \left\langle0 | ^ { \mathfrak { M } } \left\langle\left.\varkappa_{j, l}\right|^{\mathfrak{P}^{n}}\left(U_{N_{t}} \otimes \mathrm{I}^{\mathfrak{M} \mathfrak{L} \theta}\right)^{*}\right.\right.\right.\right. \\
& \left(\mathrm{I}^{\mathfrak{E}^{n}} \otimes V^{\mathfrak{Q}^{n} \mathfrak{M} \mathfrak{L} \theta}\right)^{*}\left|\zeta_{j, l, t}\right\rangle^{\mathfrak{Q}^{n} \mathfrak{E}^{n}}|j\rangle^{\mathfrak{M}}|l\rangle^{\mathfrak{L}}|t\rangle^{\theta} \\
& =F\left(\psi_{j, l, t}^{\mathfrak{Q}^{n} \mathfrak{E}^{n} \mathfrak{M} \mathfrak{L} \theta},\left|\zeta_{j, l, t}\right\rangle\left\langle\left.\zeta_{j, l, t}\right|^{\mathfrak{Q}^{n} \mathfrak{E}^{n}}\right.\right. \\
& \otimes|j\rangle\left\langle\left. j\right|^{\mathfrak{M}} \otimes \mid l\right\rangle\left\langle\left. l\right|^{\mathfrak{L}} \otimes \mid t\right\rangle\left\langle\left. t\right|^{\theta}\right) \\
& \geq 1-\epsilon .
\end{aligned}
$$

2) Building the seconder part of the decoding operator We define

$$
\left|a_{j, l}\right\rangle^{\mathfrak{P}^{n} \mathfrak{M} \mathfrak{L} \theta}:=\left|\varkappa_{j, l}\right\rangle^{\mathfrak{P}^{n}}|0\rangle^{\mathfrak{M}}|0\rangle^{\mathfrak{L}}|0\rangle^{\theta},
$$

and

$$
\begin{aligned}
& \left|b_{j, l, t}\right\rangle^{\mathfrak{P}^{n} \mathfrak{M} \mathfrak{L} \theta}:=\left(U_{N_{t}} \otimes \mathrm{I}^{\mathfrak{M} \mathfrak{L} \theta}\right)^{*}\left(\mathrm{I}^{\mathfrak{E}^{n}} \otimes V^{\mathfrak{Q}^{n} \mathfrak{M} \mathfrak{L} \theta}\right)^{*} \\
& \left|\zeta_{j, l, t}\right\rangle^{\mathfrak{Q}^{n} \mathfrak{E}^{n}}|j\rangle^{\mathfrak{M}}|l\rangle^{\mathfrak{L}}|t\rangle^{\theta} .
\end{aligned}
$$

For every $j, l$, and $t$, we have $\left\langle a_{j, l} \mid b_{j, l, t}\right\rangle^{\mathfrak{P}^{n} \mathfrak{M} \mathfrak{L} \theta} \geq 1-\epsilon$.

We define

$$
\left|\hat{a}_{j, k}\right\rangle^{\mathfrak{P}^{n} \mathfrak{M} \mathfrak{L} \theta}:=\frac{1}{\sqrt{L_{n}}} \sum_{l=1}^{L_{n}} e^{-2 \pi i l \frac{k}{L_{n}}}\left|a_{j, l}\right\rangle^{\mathfrak{P}^{n} \mathfrak{M} \mathfrak{L} \theta}
$$




$$
\left|\hat{b}_{j, k, t}\right\rangle^{\mathfrak{P}^{n} \mathfrak{M} \mathfrak{L} \theta}:=\frac{1}{\sqrt{L_{n}}} \sum_{l=1}^{L_{n}} e^{-2 \pi i l \frac{k}{L_{n}}}\left|b_{j, l, t}\right\rangle^{\mathfrak{P}^{n} \mathfrak{M} \mathfrak{L} \theta}
$$

and

$$
\left|\bar{b}_{j, k}\right\rangle^{\mathfrak{P}^{n} \mathfrak{M} \mathfrak{L} \theta}:=\frac{1}{|\theta|} \sum_{t=1}^{|\theta|}\left|\hat{b}_{j, k, t}\right\rangle^{\mathfrak{P}^{n} \mathfrak{M} \mathfrak{L} \theta} .
$$

For every $j \in\left\{1, \ldots, J_{n}\right\}$, by (81) it holds

$$
\begin{aligned}
& \frac{1}{L_{n}} \sum_{k=1}^{L_{n}}\left\langle\hat{a}_{j, k} \mid \bar{b}_{j, k}\right\rangle^{\mathfrak{P}^{n} \mathfrak{M} \mathfrak{L} \theta} \\
& =\frac{1}{|\theta|} \frac{1}{L_{n}} \sum_{t=1}^{|\theta|} \sum_{k=1}^{L_{n}}\left\langle\hat{a}_{j, k} \mid \hat{b}_{j, k, t}\right\rangle^{\mathfrak{P}^{n} \mathfrak{M} \mathfrak{L} \theta} \\
& =\frac{1}{|\theta|} \frac{1}{L_{n}} \sum_{t=1}^{|\theta|} \sum_{l=1}^{L_{n}}\left\langle a_{j, l} \mid b_{j, l, t}\right\rangle^{\mathfrak{P}^{n} \mathfrak{M} \mathfrak{L} \theta} \\
& \geq 1-\epsilon .
\end{aligned}
$$

Hence there is at least one $k_{j} \in\left\{1, \ldots, L_{n}\right\}$ such that for every $j$, we have

$$
\begin{aligned}
& 1-\epsilon \\
& \leq e^{-i s_{k_{j}}}\left\langle\hat{a}_{j, k_{j}} \mid \bar{b}_{j, k_{j}}\right\rangle^{\mathfrak{P}^{n} \mathfrak{M} \mathfrak{L} \theta} \\
& =\frac{1}{|\theta|} \sum_{t=1}^{|\theta|} e^{-i s_{k_{j}}}\left\langle\hat{a}_{j, k_{j}} \mid \hat{b}_{j, k_{j}, t}\right\rangle^{\mathfrak{P}^{n} \mathfrak{M} \mathfrak{L} \theta},
\end{aligned}
$$

for a suitable phase $s_{k_{j}}$. Since for all $t$ it holds $\left|e^{-i s_{k_{j}}}\left\langle\hat{a}_{j, k_{j}} \mid \hat{b}_{j, k_{j}, t}\right\rangle^{\mathfrak{P}^{n} \mathfrak{M} \mathfrak{L} \theta}\right| \leq 1$, we have

$$
\min _{t \in \theta}\left|e^{-i s_{k_{j}}}\left\langle\hat{a}_{j, k_{j}} \mid \hat{b}_{j, k_{j}, t}\right\rangle^{\mathfrak{P}^{n} \mathfrak{M} \mathfrak{L} \theta}\right| \geq 1-|\theta| \epsilon .
$$

Therefore, there is a suitable phase $r_{k_{j}}$ such that for all $t \in \theta$,

$$
\begin{aligned}
& 1-|\theta| \epsilon \\
& \leq\left|e^{-i s_{k_{j}}}\left\langle\hat{a}_{j, k_{j}} \mid \hat{b}_{j, k_{j}, t}\right\rangle^{\mathfrak{P}^{n} \mathfrak{M} \mathfrak{L} \theta}\right| \\
& =e^{-i r_{k_{j}}}\left\langle\hat{a}_{j, k_{j}} \mid \hat{b}_{j, k_{j}, t}\right\rangle^{\mathfrak{P}^{n} \mathfrak{M} \mathfrak{L} \theta} \\
& =e^{-i r_{k_{j}}} \frac{1}{L_{n}}\left(\sum_{l=1}^{L_{n}} e^{-2 \pi i l \frac{k_{j}}{L_{n}}}\left\langle\left. a_{j, l}\right|^{\mathfrak{P}^{n} \mathfrak{M} \mathfrak{L} \theta}\right)\right. \\
& \left(\sum_{l=1}^{L_{n}} e^{-2 \pi i l \frac{k_{j}}{L_{n}}}\left|b_{j, l, t}\right\rangle^{\mathfrak{P}^{n} \mathfrak{M} \mathfrak{L} \theta}\right) .
\end{aligned}
$$

For every $t \in \theta$, we set

$\left|\varpi_{j, t}\right\rangle^{\mathfrak{Q}^{n} \mathfrak{E}^{n} \mathfrak{L}}:=\sqrt{\frac{1}{L_{n}}} \sum_{l=1}^{L_{n}} e^{-2 \pi i\left(l \frac{k_{j}}{L_{n}}+r_{k_{j}}\right)}\left|\zeta_{j, l, t}\right|^{\mathfrak{Q}^{n} \mathfrak{E}^{n}} \otimes|l\rangle^{\mathfrak{L}}$ and

$$
\begin{aligned}
& \left|\vartheta_{j, t}\right\rangle^{\mathfrak{Q}^{n} \mathfrak{E}^{n} \mathfrak{M} \mathfrak{L} \theta}:=\sqrt{\frac{1}{L_{n}}} \sum_{l=1}^{L_{n}} e^{-2 \pi i l \frac{k_{j}}{L_{n}}}\left[\mathrm{I}^{\mathfrak{E}^{n}} \otimes V^{\mathfrak{Q}^{n} \mathfrak{M} \mathfrak{L} \theta}\right] \\
& \left(U_{N}^{n}\left|\varkappa_{j, l}\right\rangle^{\mathfrak{P}^{n}}\right)|0\rangle^{\mathfrak{M}}|0\rangle^{\mathfrak{L}}|0\rangle^{\theta} .
\end{aligned}
$$

For all $t \in \theta$ and $j \in\left\{1, \ldots J_{n}\right\}$ it holds by (83)

$$
\begin{aligned}
& F\left(| \vartheta _ { j , t } \rangle \left\langle\left.\vartheta_{j, t}\right|^{\mathfrak{Q}^{n} \mathfrak{E}^{n} \mathfrak{M} \mathfrak{L} \theta},\right.\right. \\
& \left|\varpi_{j, t}\right\rangle\left\langle\left.\varpi_{j, t}\right|^{\mathfrak{Q}^{n} \mathfrak{E}^{n} \mathfrak{L}} \otimes \mid j\right\rangle\left\langle\left. j\right|^{\mathfrak{M}} \otimes \mid t\right\rangle\left\langle\left. t\right|^{\theta}\right) \\
& \left.=\left|\left\langle\left.\vartheta_{j, t}\right|^{\mathfrak{Q}^{n} \mathfrak{E}^{n} \mathfrak{M} \mathfrak{L} \theta} \mid \varpi_{j, t}\right\rangle^{\mathfrak{Q}^{n} \mathfrak{E}^{n} \mathfrak{L}}\right| j\right\rangle^{M}|t\rangle^{\theta} \mid \\
& =\frac{1}{L_{n}}\left(\sum_{l=1}^{L_{n}} e^{-2 \pi i l \frac{k_{j}}{L_{n}}}\left\langle\left. a_{j, l}\right|^{\mathfrak{P}^{n} \mathfrak{M} \mathfrak{L} \theta}\right)\right. \\
& \left(\sum_{l=1}^{L_{n}} e^{-2 \pi i l} \frac{k_{j}}{L_{n}} e^{-i r_{k_{j}}}\left|b_{j, l, t}\right\rangle^{\mathfrak{P}^{n} \mathfrak{M} \mathfrak{L} \theta}\right) \\
& \geq 1-|\theta| \epsilon .
\end{aligned}
$$

Furthermore, since (79) holds there is a quantum state $\xi_{t}^{\mathfrak{E}^{n}}$, which does not depend on $j$ and $l$, on $H^{\mathfrak{E}^{n}}$ such that

$$
\left\|\xi_{t}^{\mathfrak{E}^{n}}-\operatorname{tr}_{\mathfrak{Q}^{n}}\left(U_{N_{t}}\left|\varkappa_{j, l}\right\rangle\left\langle\left.\varkappa_{j, l}\right|^{\mathfrak{P}^{n}} U_{N_{t}}^{*}\right) \|_{1} \leq \epsilon .\right.\right.
$$

By monotonicity of fidelity, for any $l \in\left\{1, \ldots, L_{n}\right\}$

$$
\begin{aligned}
& \left\|\operatorname { t r } _ { \mathfrak { Q } ^ { n } } \left(U_{N_{t}}\left|\varkappa_{j, l}\right\rangle\left\langle\left.\varkappa_{j, l}\right|^{\mathfrak{P}^{n}} U_{N_{t}}^{*}\right)-\operatorname{tr}_{\mathfrak{Q}^{n}}\left(\left|\zeta_{j, l, t}\right\rangle\left\langle\left.\zeta_{j, l, t}\right|^{\mathfrak{Q}^{n} \mathfrak{E}^{n}}\right) \|_{1}\right.\right.\right. \\
& \leq 2\left[1-F\left(\operatorname { t r } _ { \mathfrak { Q } ^ { n } } \left(U_{N_{t}}\left|\varkappa_{j, l}\right\rangle\left\langle\left.\varkappa_{j, l}\right|^{\mathfrak{P}^{n}} U_{N_{t}}^{*}\right)\right.\right.\right. \\
& \left.\operatorname{tr}_{\mathfrak{Q}^{n}}\left(\left|\zeta_{j, l, t}\right\rangle\left\langle\left.\zeta_{j, l, t}\right|^{\mathfrak{Q}^{n} \mathfrak{E}^{n}}\right)\right)\right]^{\frac{1}{2}} \\
& \leq 2\left[1-F\left(\psi_{j, l, t}^{\mathfrak{Q}^{n} \mathfrak{E}^{n} \mathfrak{M} \mathfrak{N} \theta},\left|\zeta_{j, l, t}\right\rangle\left\langle\left.\zeta_{j, l, t}\right|^{\mathfrak{Q}^{n} \mathfrak{E}^{n}}\right.\right.\right. \\
& \left.\otimes|j\rangle\left\langle\left. j\right|^{\mathfrak{M}} \otimes \mid l\right\rangle\left\langle\left. l\right|^{\mathfrak{L}} \otimes \mid t\right\rangle\left\langle\left. t\right|^{\theta}\right)\right]^{\frac{1}{2}} \\
& \leq 2 \sqrt{\epsilon}
\end{aligned}
$$

the first inequality holds because for two quantum states $\varrho$ and $\eta$, we have $\frac{1}{2}\|\varrho-\eta\|_{1} \leq \sqrt{1-F(\varrho, \eta)^{2}}$.

By (85) and (86)

$$
\begin{aligned}
& \left\|\operatorname { t r } _ { \mathfrak { Q } ^ { n } \mathfrak { L } } \left(\left|\varpi_{j, t}\right\rangle\left\langle\left.\varpi_{j, t}\right|^{\mathfrak{Q}^{n} \mathfrak{E}^{n} \mathfrak{L}}\right)-\xi_{t}^{\mathfrak{E}^{n}} \|_{1}\right.\right. \\
& =\left\|\frac { 1 } { L _ { n } } \sum _ { l = 1 } ^ { L _ { n } } \operatorname { t r } _ { \mathfrak { Q } ^ { n } } \left(\left|\zeta_{j, l, t}\right\rangle\left\langle\left.\zeta_{j, l, t}\right|^{\mathfrak{Q}^{n} \mathfrak{E}^{n}}\right)-\xi_{t}^{\mathfrak{E}^{n}} \|_{1}\right.\right. \\
& \leq \frac{1}{L_{n}} \sum_{l=1}^{L_{n}} \| \operatorname{tr}_{\mathfrak{Q}^{n}}\left(U_{N_{t}}\left|\varkappa_{j, l}\right\rangle\left\langle\left.\varkappa_{j, l}\right|^{\mathfrak{P}^{n}} U_{N_{t}}^{*}\right)\right. \\
& -\operatorname{tr}_{\mathfrak{Q}^{n}}\left(\left|\zeta_{j, l, t}\right\rangle\left\langle\left.\zeta_{j, l, t}\right|^{\mathfrak{Q}^{n} \mathfrak{E}^{n}}\right) \|_{1}\right. \\
& +\left\|\xi_{t}^{\mathfrak{E}^{n}}-\operatorname{tr}_{\mathfrak{Q}^{n}}\left(U_{N_{t}}\left|\varkappa_{j, l}\right\rangle\left\langle\left.\varkappa_{j, l}\right|^{\mathfrak{P}^{n}} U_{N_{t}}^{*}\right) \|_{1}\right.\right. \\
& \leq 2 \sqrt{\epsilon}+\epsilon
\end{aligned}
$$


holds for all $t \in \theta$ and $j \in\left\{1, \ldots, J_{n}\right\}$.

In 36] (cf. also 20]) it was shown that when (87) holds, for every $t \in \theta$ we can find a unitary operator $U_{(t)}^{\mathfrak{Q}^{n} \mathfrak{M} \mathfrak{L}}$ such that if we set

$$
\begin{aligned}
& \chi_{j, j^{\prime}, t}^{\mathfrak{Q}^{n} \mathfrak{E}^{n} \mathfrak{M} \mathfrak{L}}:=\left(U_{(t)}^{\mathfrak{Q}^{n} \mathfrak{M} \mathfrak{L}} \otimes \mathrm{I}^{\mathfrak{E}^{n}}\right) \\
& \left(\left|\varpi_{j, t}\right\rangle\left\langle\left.\varpi_{j, t}\right|^{\mathfrak{Q}^{n} \mathfrak{E}^{n} \mathfrak{L}} \otimes \mid j\right\rangle\left\langle\left. j^{\prime}\right|^{\mathfrak{M}}\right)\left(U_{(t)}^{\mathfrak{Q}^{n} \mathfrak{M} \mathfrak{L}} \otimes \mathrm{I}^{\mathfrak{E}^{n}}\right)^{*},\right.
\end{aligned}
$$

then

$$
F\left(\left|\xi_{t}\right\rangle\left\langle\left.\xi_{t}\right|^{\mathfrak{Q}^{n} \mathfrak{E}^{n} \mathfrak{L}} \otimes \mid j\right\rangle\left\langle\left. j^{\prime}\right|^{\mathfrak{M}}, \chi_{j, j^{\prime}, t}^{\mathfrak{Q}^{n} \mathfrak{E}^{n}} \mathfrak{M} \mathfrak{L}\right) \geq 1-4 \epsilon-4 \sqrt{\epsilon},\right.
$$

where $\left|\xi_{t}\right|^{\mathfrak{Q}^{n} \mathfrak{E}^{n} \mathfrak{L}}$ is chosen so that $\left|\xi_{t}\right\rangle\left\langle\left.\xi_{t}\right|^{\mathfrak{Q}^{n} \mathfrak{E}^{n} \mathfrak{L}}\right.$ is a purification of $\xi_{t}^{\mathfrak{E}^{n}}$ on $H^{\mathfrak{Q}^{n} \mathfrak{E}^{n} \mathfrak{L}}$.

\section{3) Defining the code}

We can now define our entanglement generating code. Let $t^{\prime}$ be arbitrary in $\theta$. The sender prepares the quantum state

$$
\begin{aligned}
& \frac{1}{J_{n}} \frac{1}{L_{n}}\left(\sum_{j=1}^{J_{n}} \sum_{l=1}^{L_{n}} e^{-2 \pi i l} \frac{k_{j}}{L_{n}}\left|\varkappa_{j, l}\right\rangle^{\mathfrak{P}^{n}}|j\rangle^{\mathfrak{A}}\right) \\
& \left(\sum_{j=1}^{J_{n}} \sum_{l=1}^{L_{n}} e^{-2 \pi i l \frac{k_{j}}{L_{n}}\left\langle\left. j\right|^{\mathfrak{A}}\left\langle\left.\varkappa_{j, l}\right|^{\mathfrak{P}^{n}}\right)\right.},\right.
\end{aligned}
$$

keeps the system $\mathfrak{A}$, and sends the system $\mathfrak{P}^{n}$ through the channel $N_{t^{\prime}}^{\otimes n}$, i.e., the resulting quantum state is

$$
\begin{aligned}
& \frac{1}{J_{n}} \frac{1}{L_{n}}\left(\mathrm{I}^{\mathfrak{A}} \otimes U_{N_{t^{\prime}}}^{n}\right)\left[\left(\sum_{j=1}^{J_{n}} \sum_{l=1}^{L_{n}} e^{-2 \pi i l \frac{k_{j}}{L_{n}}}|j\rangle^{A}\left|\varkappa_{j, l}\right\rangle^{\mathfrak{P}^{n}}\right)\right. \\
& \left(\sum_{j=1}^{J_{n}} \sum_{l=1}^{L_{n}} e^{-2 \pi i l \frac{k_{j}}{L_{n}}}\left\langle\left.\varkappa_{j, l}\right|^{\mathfrak{P}^{n}}\left\langle\left. j\right|^{\mathfrak{A}}\right)\right]\left(\mathrm{I}^{\mathfrak{A}} \otimes U_{N_{t^{\prime}}}^{n}\right)^{*}\right. \\
& =\frac{1}{J_{n}} \frac{1}{L_{n}}\left[\sum_{j=1}^{J_{n}}|j\rangle^{\mathfrak{A}}\left(\sum_{l=1}^{L_{n}} e^{-2 \pi i l} \frac{k_{j}}{L_{n}} U_{N_{t^{\prime}}}^{n}\left|\varkappa_{j, l}\right\rangle^{\mathfrak{P}^{n}}\right)\right] \\
& {\left[\sum _ { j = 1 } ^ { J _ { n } } \left(\sum_{l=1}^{L_{n}} e^{-2 \pi i l \frac{k_{j}}{L_{n}}}\left\langle\left.\varkappa_{j, l}\right|^{\mathfrak{P}^{n}}\left(U_{N_{t^{\prime}}}^{n}\right)^{*}\right)\left\langle\left. j\right|^{\mathfrak{A}}\right] .\right.\right.}
\end{aligned}
$$

The receiver subsequently applies the decoding operator

$$
\begin{gathered}
\tau^{\mathfrak{Q}^{n}} \rightarrow \operatorname{tr}_{\mathfrak{Q}^{n} \mathfrak{L} \theta}\left[\left(\sum_{t \in \theta} U_{(t)}^{\mathfrak{Q}^{n} \mathfrak{M} \mathfrak{L}} \otimes|t\rangle\left\langle\left. t\right|^{\theta}\right) V^{\mathfrak{Q}^{n} \mathfrak{M} \mathfrak{L} \theta}\right.\right. \\
\left(\tau^{\mathfrak{Q}^{n}} \otimes|0\rangle\left\langle\left. 0\right|^{\mathfrak{M}} \otimes \mid 0\right\rangle\left\langle\left. 0\right|^{\mathfrak{L}} \otimes \mid 0\right\rangle\left\langle\left. 0\right|^{\theta}\right)\right. \\
V^{\mathfrak{Q}^{n} \mathfrak{M} \mathfrak{L} \theta^{*}}\left(\sum_{t \in \theta} U_{(t)}^{\mathfrak{Q}^{n} \mathfrak{M} \mathfrak{L}} \otimes|t\rangle\left\langle\left. t\right|^{\theta}\right)^{*}\right],
\end{gathered}
$$

to his outcome.

3.1) The resulting quantum state after performing the decoding operator
We define

$\iota_{t^{\prime}}^{\mathfrak{A} \mathfrak{Q}^{n} \mathfrak{E}^{n} \mathfrak{M} \mathfrak{L} \theta}$

$$
\begin{aligned}
& :=\left(\sum_{t \in \theta} U_{(t)}^{\mathfrak{Q}^{n} \mathfrak{M} \mathfrak{L}} \otimes \mathrm{I}^{\mathfrak{A} \mathfrak{E}^{n}} \otimes|t\rangle\left\langle\left. t\right|^{\theta}\right)\left(V^{\mathfrak{Q}^{n} \mathfrak{M} \mathfrak{L} \theta} \otimes \mathrm{I}^{\mathfrak{A} \mathfrak{E}^{n}}\right)\right. \\
& \left(\frac{1}{J_{n}} \frac{1}{L_{n}}\left[\sum_{j=1}^{J_{n}}|j\rangle^{\mathfrak{A}}\left(\sum_{l=1}^{L_{n}} e^{-2 \pi i l} \frac{k_{j}}{L_{n}} U_{N_{t^{\prime}}}^{n}\left|\varkappa_{j, l}\right\rangle^{P^{n}}\right)\right]\right. \\
& {\left[\sum _ { j = 1 } ^ { J _ { n } } \left(\sum_{l=1}^{L_{n}} e^{-2 \pi i l} \frac{k_{j}}{L_{n}}\left\langle\left.\varkappa_{j, l}\right|^{P^{n}}\left(U_{N_{t^{\prime}}}^{n}\right)^{*}\right)\left\langle\left. j\right|^{\mathfrak{A}}\right]\right.\right.} \\
& \otimes|0\rangle\left\langle\left. 0\right|^{\mathfrak{M}} \otimes \mid 0\right\rangle\left\langle\left. 0\right|^{\mathfrak{L}} \otimes \mid 0\right\rangle\left\langle\left. 0\right|^{\theta}\right)\left(V^{\mathfrak{Q}^{n} \mathfrak{M} \mathfrak{L} \theta} \otimes \mathrm{I}^{\mathfrak{A} \mathfrak{E}^{n}}\right)^{*} \\
& \left(\sum_{t \in \theta} U_{(t)}^{\mathfrak{Q}^{n} \mathfrak{M} \mathfrak{L}} \otimes \mathrm{I}^{\mathfrak{A L E}} \otimes|t\rangle\left\langle\left. t\right|^{\theta}\right)^{*}\right. \\
& =\left(\sum_{t \in \theta} U_{(t)}^{\mathfrak{Q}^{n} \mathfrak{M} \mathfrak{L}} \otimes \mathrm{I}^{\mathfrak{A} \mathfrak{E}^{n}} \otimes|t\rangle\left\langle\left. t\right|^{\theta}\right)\right. \\
& \left(\frac { 1 } { J _ { n } } ( \sum _ { j = 1 } ^ { J _ { n } } | j \rangle ^ { \mathfrak { A } } | \vartheta _ { j , t ^ { \prime } } \rangle ^ { \mathfrak { Q } ^ { n } \mathfrak { E } ^ { n } \mathfrak { M } \mathfrak { L } \theta } ) \left(\sum_{j=1}^{J_{n}}\left\langle\left.\vartheta_{j, t^{\prime}}\right|^{\mathfrak{Q}^{n} \mathfrak{E}^{n} \mathfrak{M} \mathfrak{L} \theta}\left\langle\left. j\right|^{\mathfrak{A}}\right)\right)\right.\right. \\
& \left(\sum_{t \in \theta} U_{(t)}^{\mathfrak{Q}^{n} \mathfrak{M} \mathfrak{L}} \otimes \mathrm{I}^{\mathfrak{A} \mathfrak{E} \mathfrak{E}^{n}} \otimes|t\rangle\left\langle\left. t\right|^{\theta}\right)^{*},\right.
\end{aligned}
$$

then the resulting quantum state after performing the decoding operator is $\operatorname{tr}_{\mathfrak{Q}^{n} \mathfrak{E} n} \mathfrak{L} \theta\left(\iota_{t^{\prime}}^{\mathfrak{A} \mathfrak{Q}^{n} \mathfrak{E}^{n} \mathfrak{M} \mathfrak{L} \theta}\right)$.

3.2) The fidelity of $\frac{1}{J_{n}} \sum_{j=1}^{J_{n}} \sum_{j^{\prime}=1}^{J_{n}} \chi_{j, j^{\prime}, t^{\prime}}^{\mathfrak{Q}^{n} \mathfrak{E}^{n}} \mathfrak{M} \mathfrak{L} \otimes|j\rangle\left\langle\left. j\right|^{\mathfrak{A}} \otimes\right.$ $\left|t^{\prime}\right\rangle\left\langle\left. t^{\prime}\right|^{\theta}\right.$ and the actual quantum state

$$
\begin{aligned}
& \left(\sum_{t \in \theta} U_{(t)}^{\mathfrak{Q}^{n} \mathfrak{M} \mathfrak{L}} \otimes \mathrm{I}^{\mathfrak{A} \mathfrak{E}^{n}} \otimes|t\rangle\left\langle\left. t\right|^{\theta}\right)\right. \\
& \left(\sum_{t \in \theta} U_{(t)}^{\mathfrak{Q}^{n} \mathfrak{M} \mathfrak{L}} \otimes \mathrm{I}^{\mathfrak{E}^{n}} \otimes|t\rangle\left\langle\left. t\right|^{\theta}\right)^{*}\right. \\
& =\mathrm{I}^{\mathfrak{A} \mathfrak{E}} \otimes \sum_{t \in \theta} U_{(t)}^{\mathfrak{Q}^{n} \mathfrak{M} \mathfrak{L}}\left(U_{(t)}^{\mathfrak{Q}^{n}} \mathfrak{M} \mathfrak{L}\right)^{*} \otimes|t\rangle\left\langle\left. t\right|^{\theta}\right. \\
& =\mathrm{I}^{\mathfrak{A} \mathfrak{Q}^{n} \mathfrak{E}^{n} \mathfrak{M} \mathfrak{L} \theta},
\end{aligned}
$$

$\sum_{t \in \theta} U_{(t)}^{\mathfrak{Q}^{n} \mathfrak{M} \mathfrak{L}} \otimes \mathrm{I}^{\mathfrak{E}^{n}} \otimes|t\rangle\left\langle\left. t\right|^{\theta}\right.$ is unitary.

Because of this unitarity and by (84)

$$
\begin{aligned}
& F\left(\iota_{t^{\prime}}^{\mathfrak{Q} \mathfrak{Q}^{n} \mathfrak{E}^{n} \mathfrak{M} \mathfrak{L} \theta}, \frac{1}{J_{n}} \sum_{j=1}^{J_{n}} \sum_{j^{\prime}=1}^{J_{n}} \chi_{j, j^{\prime}, t^{\prime}}^{\mathfrak{Q}^{n} \mathfrak{E}^{n} \mathfrak{M} \mathfrak{L}} \otimes|j\rangle\left\langle\left. j^{\prime}\right|^{\mathfrak{A}} \otimes \mid t^{\prime}\right\rangle\left\langle\left. t^{\prime}\right|^{\theta}\right)\right. \\
& =F\left(\frac { 1 } { J _ { n } } ( \sum _ { j = 1 } ^ { J _ { n } } | j \rangle ^ { \mathfrak { A } } | \vartheta _ { j , t ^ { \prime } } \rangle ^ { \mathfrak { Q } ^ { n } \mathfrak { E } ^ { n } \mathfrak { M } \mathfrak { L } \theta } ) \left(\sum _ { j = 1 } ^ { J _ { n } } \left\langle\left.\vartheta_{j, t^{\prime}}\right|^{\mathfrak{Q}^{n} \mathfrak{E} \mathfrak{M} \mathfrak{M} \theta}\left\langle\left. j\right|^{\mathfrak{A}}\right),\right.\right.\right. \\
& \frac{1}{J_{n}}\left(\sum_{j=1}^{J_{n}}\left|\varpi_{j, t^{\prime}}\right\rangle^{\mathfrak{Q}^{n} \mathfrak{E}^{n} \mathfrak{L}} \otimes|j\rangle^{\mathfrak{A}} \otimes|j\rangle^{\mathfrak{M}}\right)
\end{aligned}
$$




$$
\begin{aligned}
& \left(\sum _ { j = 1 } ^ { J _ { n } } \left\langle\left.j\right|^{\mathfrak{M}} \otimes\left\langle\left. j\right|^{\mathfrak{A}} \otimes\left\langle\left.\varpi_{j, t^{\prime}}\right|^{\mathfrak{Q}^{n} \mathfrak{E}^{n} \mathfrak{L}}\right) \otimes \mid t^{\prime}\right\rangle\left\langle\left. t^{\prime}\right|^{\theta}\right)\right.\right. \\
& =\frac{1}{J_{n}} \mid\left(\sum_{j=1}^{J_{n}}\left\langle\left.\vartheta_{j, t^{\prime}}\right|^{\mathfrak{Q}^{n} \mathfrak{E}^{n} \mathfrak{M} \mathfrak{L} \theta}\right)\right. \\
& \left(\sum_{j=1}^{J_{n}}\left|\varpi_{j, t^{\prime}}\right\rangle^{\mathfrak{Q}^{n} \mathfrak{E}^{n} \mathfrak{L}} \otimes|j\rangle^{\mathfrak{M}} \otimes\left|t^{\prime}\right\rangle^{\theta}\right) \mid \\
& \geq 1-|\theta| \epsilon .
\end{aligned}
$$

3.3) The fidelity of $\frac{1}{J_{n}} \sum_{j=1}^{J_{n}} \sum_{j^{\prime}=1}^{J_{n}} \chi_{j, j^{\prime}, t^{\prime}}^{\mathfrak{Q}^{n} \mathfrak{E}^{n}} \mathfrak{M L} \otimes|j\rangle\left\langle\left. j^{\prime}\right|^{\mathfrak{A}} \otimes\right.$ $\left|t^{\prime}\right\rangle\left\langle\left. t^{\prime}\right|^{\theta}\right.$ and the standard maximally entanglement state

By (88) we have

$$
\begin{aligned}
& F\left(\frac { 1 } { J _ { n } } \sum _ { j = 1 } ^ { J _ { n } } \sum _ { j ^ { \prime } = 1 } ^ { J _ { n } } \chi _ { j , j ^ { \prime } , t ^ { \prime } } ^ { \mathfrak { Q } ^ { n } \mathfrak { E } ^ { n } \mathfrak { M } \mathfrak { L } } \otimes | t ^ { \prime } \rangle \langle t ^ { \prime } | ^ { \theta } \otimes | j \rangle \left\langle\left.j^{\prime}\right|^{\mathfrak{A}},\right.\right. \\
& \frac{1}{J_{n}}\left(\sum_{j=1}^{J_{n}}\left|\xi_{t^{\prime}}\right\rangle^{\mathfrak{Q}^{n} \mathfrak{E}^{n} \mathfrak{L}} \otimes|j\rangle^{\mathfrak{A}} \otimes|j\rangle^{\mathfrak{M}} \otimes\left|t^{\prime}\right\rangle^{\theta}\right) \\
& \left(\sum _ { j = 1 } ^ { J _ { n } } \left\langle\xi _ { t ^ { \prime } } | ^ { \mathfrak { Q } ^ { n } \mathfrak { E } ^ { n } \mathfrak { L } } \otimes \left\langle\left.j\right|^{\mathfrak{A}} \otimes\left\langle\left. j\right|^{\mathfrak{M}} \otimes\left\langle\left. t^{\prime}\right|^{\theta}\right)\right)\right.\right.\right. \\
& \geq 1-4 \epsilon-4 \sqrt{\epsilon} .
\end{aligned}
$$

3.4) The fidelity of the actual quantum state and the standard maximally entanglement state

Since for two quantum states $\varrho$ and $\eta$, it holds

$$
1-F(\varrho, \eta) \leq \frac{1}{2}\|\varrho-\eta\|_{1} \leq \sqrt{1-F(\varrho, \eta)^{2}},
$$

for three quantum states $\varrho, \eta$, and $v$, we have

$$
\begin{aligned}
& F(\varrho, \eta) \\
& \geq 1-\frac{1}{2}\|\varrho-\eta\|_{1} \\
& \geq 1-\frac{1}{2}\|\varrho-v\|_{1}-\frac{1}{2}\|v-\eta\|_{1} \\
& \geq 1-\sqrt{1-F(\varrho, v)^{2}}-\sqrt{1-F(v, \eta)^{2}} .
\end{aligned}
$$

Combining (92) and (93), for all $t^{\prime} \in \theta$ we have

$$
\begin{aligned}
& F\left(\operatorname{tr}_{\mathfrak{Q}^{n} \mathfrak{E} n \mathfrak{L} \theta}\left(\iota_{t^{\prime}}^{\mathfrak{A} \mathfrak{Q}^{n} \mathfrak{E}^{n} \mathfrak{M} \mathfrak{L} \theta}\right),\right. \\
& \left(\sum_{j=1}^{J_{n}}|j\rangle^{\mathfrak{A}} \otimes|j\rangle^{\mathfrak{M}}\right)\left(\sum_{j=1}^{J_{n}}\left\langle\left. j\right|^{\mathfrak{A}} \otimes\left\langle\left. j\right|^{\mathfrak{M}}\right)\right)\right. \\
& \geq F\left(\iota_{t^{\prime}}^{\mathfrak{A} \mathfrak{Q}^{n} \mathfrak{E}^{n} \mathfrak{M} \mathfrak{L} \theta}, \frac{1}{J_{n}}\left(\sum_{j=1}^{J_{n}}\left|\xi_{t^{\prime}}\right\rangle^{\mathfrak{Q}^{n} \mathfrak{E}^{n} \mathfrak{L}} \otimes|j\rangle^{\mathfrak{A}} \otimes|j\rangle^{\mathfrak{M}} \otimes\left|t^{\prime}\right\rangle^{\theta}\right)\right. \\
& \left(\sum _ { j = 1 } ^ { J _ { n } } \left\langle\xi _ { t ^ { \prime } } | ^ { \mathfrak { Q } ^ { n } \mathfrak { E } ^ { n } \mathfrak { L } } \otimes \left\langle\left.j\right|^{\mathfrak{A}} \otimes\left\langle\left. j\right|^{\mathfrak{M}} \otimes\left\langle\left. t^{\prime}\right|^{\theta}\right)\right)\right.\right.\right.
\end{aligned}
$$

$$
\begin{aligned}
& \geq 1-\sqrt{2|\theta| \epsilon-|\theta|^{2} \epsilon^{2}}-\sqrt{8 \sqrt{\epsilon}-16 \epsilon^{2}-32 \epsilon \sqrt{\epsilon}-8 \epsilon} \\
& \geq 1-\sqrt{2|\theta|} \sqrt{\epsilon}-\sqrt{8} \sqrt[4]{\epsilon} .
\end{aligned}
$$

This means that if $n$ is large enough, then for any positive $\delta$ and $\epsilon$, there is an $(n, \sqrt{2|\theta|} \sqrt{\epsilon}+\sqrt{8} \sqrt[4]{\epsilon})$ code with rate

$$
\min _{t} \chi\left(X ; Q_{t}\right)-\max _{t} \chi\left(X ; E_{t}\right)-2 \delta .
$$

Proposition 1 The entanglement generating capacity of $\left(N_{t}\right)_{t \in \theta}$ with CSI at the encoder is

$$
A_{C S I}=\lim _{n \rightarrow \infty} \frac{1}{n} \min _{t \in \theta} \max _{\rho \in \mathcal{S}(H)^{\mathfrak{Q}^{n}}} I_{C}\left(\rho ; N_{t}^{\otimes n}\right) .
$$

Proof. As the authors of [18] showed, after receiving a dummy code word as the first block, the receiver also can have CSI. Then we have the case where both the sender and the receiver have CSI. But this case is equivalent to the case where we only have one channel $\left(N_{t}\right)$ instead of a family of channels $\left\{\left(N_{t}\right): t=1, \ldots,|\theta|\right\}$, and we may assume it is the worst channel. The bits that we use to detect the CSI are large but constant, so it is negligible compared to the rest. By [20], the entanglement generating capacity of the quantum channel $N_{t}$ is

$$
\lim _{n \rightarrow \infty} \frac{1}{n} \max _{\rho \in \mathcal{S}(H)^{\mathfrak{Q}^{n}}} I_{C}\left(\rho ; N_{t}^{\otimes n}\right) .
$$

The proof of the converse is similar to those given in the proof of Theorem 2, where we consider a worst $t^{\prime}$.

Proposition 2 The entanglement generating capacity of $\left(N_{t}\right)_{t \in \theta}$ with feedback is bounded as follows

$$
A_{\text {feed }} \geq \lim _{n \rightarrow \infty} \frac{1}{n} \min _{t \in \theta} \max _{\rho \in \mathcal{S}(H)^{2^{n}}} I_{C}\left(\rho ; N_{t}^{\otimes n}\right) .
$$

Proof. As the authors of [18] showed, the receiver can detect the channel state $t$ correctly after receiving a dummy word as the first block. Then he can send $t$ back to the sender via feedback.

Remark 8 Feedback can improve the channel capacity of quantum channels in some cases (c.f. [27]). Thus it can be possible that the lower bound in Proposition Q is not tight. For a one-way entanglement distillation protocol using secret key, cf. [21]. 


\section{FURTHER NOTES}

In this section we will discuss the proof of our result of the previous section.

Let $\mathfrak{P}, \mathfrak{Q}, H^{\mathfrak{P}}$, and $H^{\mathfrak{Q}}$ be defined as in Section II Let $N$ be a quantum channel $\mathcal{S}\left(H^{\mathfrak{P}}\right) \rightarrow \mathcal{S}\left(H^{\mathfrak{Q}}\right)$. In general, there are two ways to represent a quantum channel, i. e. a completely positive trace preserving map $\mathcal{S}\left(H^{\mathfrak{P}}\right) \rightarrow$ $\mathcal{S}\left(H^{\mathfrak{Q}}\right)$, with linear algebraic tools.

\section{Operator Sum Decomposition (Kraus Representation)}

$$
N(\rho)=\sum_{i=1}^{K} A_{i} \rho A_{i}{ }^{*}
$$

where $A_{1}, \ldots, A_{K}$ (Kraus operators) are linear operators $\mathcal{S}\left(H^{\mathfrak{P}}\right) \rightarrow \mathcal{S}\left(H^{\mathfrak{Q}}\right)$ (cf. [26], [6], and [31]). They satisfy the completeness relation $\sum_{i=1}^{K} A_{i}{ }^{*} A_{i}=\mathrm{I}_{H^{\mathfrak{P}}}$. The representation of a quantum channel $N$ according to (98) is not unique. Let $A_{1}, \ldots, A_{K}$ and $B_{1}, \ldots, B_{K^{\prime}}$ be two sets of Kraus operators (by appending zero operators to the shorter list of operation elements we may ensure that $\left.K^{\prime}=K\right)$. Suppose $A_{1}, \ldots, A_{K}$ represents $N$, then $B_{1}, \ldots, B_{K}$ also represents $N$ if and only if there exist a $K \times K$ unitary matrix $\left(u_{i, j}\right)_{i, j=1, \ldots, K}$ such that for all $i$ we have $A_{i}=\sum_{j=1}^{K} u_{i, j} B_{j}$ (cf. 31]).

\section{Isometric Extension (Stinespring Dilation)}

$$
N(\rho)=\operatorname{tr}_{\mathfrak{E}}\left(U_{N} \rho U_{N}^{*}\right)
$$

where $U_{N}$ is a linear operator $\mathcal{S}\left(H^{\mathfrak{P}}\right) \rightarrow \mathcal{S}\left(H^{\mathfrak{Q E}}\right)$ such that $U_{N}^{*} U_{N}=\mathrm{I}_{H^{\mathfrak{P}}}$, and $\mathfrak{E}$ is the quantum system of the environment (cf. 37], 6], and also cf. 38] for a more general Stinespring Dilation Theorem). $H^{\mathfrak{E}}$ can be chosen such that $\operatorname{dim} H^{\mathfrak{E}} \leq\left(\operatorname{dim} H^{\mathfrak{P}}\right)^{2}$. The isometric extension of a quantum channel $N$ according to (99) is not unique either. Let $U$ and $U^{\prime}$ be two linear operators $\mathcal{S}\left(H^{\mathfrak{P}}\right) \rightarrow \mathcal{S}\left(H^{\mathfrak{Q E}}\right)$. Suppose $U$ represents $N$, then $U^{\prime}$ also represents $N$ if and only if $U$ and $U^{\prime}$ are unitarily equivalent.

It is well known that we can reduce each of these two representations of the quantum channel from the other one. Let $A_{1}, \ldots, A_{K}$ be a set of Kraus operators which represents $N$. Let $\left\{|j\rangle^{\mathfrak{E}}: j=1, \ldots, K\right\}$ be an orthonormal system on $H^{\mathfrak{E}}$. Then $U_{N}=\sum_{j=1}^{K} A_{j} \otimes|j\rangle^{\mathfrak{E}}$ is an isometric extension which represents $N$, since $\left(\sum_{j=1}^{K} A_{j} \otimes|j\rangle^{\mathfrak{E}}\right)$ $\rho \quad\left(\sum_{k=1}^{K} A_{k} \otimes|k\rangle^{\mathfrak{E}}\right)^{*}=\sum_{j=1}^{K} A_{j} \rho A_{j}{ }^{*} \quad$ and $\left(\sum_{j=1}^{K} A_{j} \otimes|j\rangle^{\mathfrak{E}}\right)^{*}\left(\sum_{k=1}^{K} A_{k} \otimes|k\rangle^{\mathfrak{E}}\right)=\sum_{j=1}^{K} A_{j}{ }^{*} A_{j}$. For the other way around, every isometric extension $U_{N}$ that represents $N$ can be written in the form $U_{N}=\sum_{j=1}^{K} A_{j} \otimes|j\rangle^{\mathfrak{E}}$, i.e. if the sender sends $\rho$, and if the environment's measurement gives $|i\rangle^{\mathfrak{E}}$, the receiver's outcome will be $A_{i} \rho A_{i}{ }^{*}$. Here $A_{1}, \ldots, A_{K}$ is a set of Kraus operators which represents $N$, and $\left\{|j\rangle^{\mathfrak{E}}: j=1, \ldots, K\right\}$ is an orthonormal system on $H^{\mathfrak{E}}$.

Using either of both methods to represent a quantum channel, one can show that (cf. [20]) the entanglement generating capacity of a quantum channel $N$ is

$$
\mathcal{A}(N)=\lim _{n \rightarrow \infty} \frac{1}{n} \max _{\rho \in \mathcal{S}(H)^{\mathfrak{Q}^{n}}} I_{C}\left(\rho ; N^{\otimes n}\right) .
$$

The Kraus representation describes the dynamics of the principal system without having to explicitly consider properties of the environment, whose dynamics are often unimportant. All that we need to know is the system of the receiver alone; this simplifies calculations. In [25], an explicit construction of a quantum error correction code (both perfect and approximate information recovery) with the Kraus operators is given. In the Stinespring dilation, we have a natural interpretation of the system of the environment. From the Stinespring dilation, we can conclude that the receiver can detect almost all quantum information if and only if the channel releases almost no information to the environment. In [36], an alternative way to build a quantum error correction code (both perfect and approximate information recovery) is given using this fact. The disadvantage is that we suppose it is suboptimal for calculating the entanglement generating capacity of a compound quantum channel without CSI at the encoder.

In [12], the entanglement generating capacity for the compound quantum channel is determined, using a quantum error correction code of 25], which is built by Kraus operators. Their result is the following. The entanglement generating capacity of a quantum wiretap channel $N=\left(N_{t}\right)_{t \in \theta}$ is

$$
\mathcal{A}(N)=\lim _{n \rightarrow \infty} \frac{1}{n} \max _{\rho \in \mathcal{S}(H)^{\mathfrak{Q}^{n}}} \min _{t \in \theta} I_{C}\left(\rho ; N_{t}^{\otimes n}\right)
$$

This result is stronger than our result in Theorem 3 . This is due to the fact that we use for our proof a quantum error correction code of [36], which is based upon the Stinespring dilation. If we use the Kraus operators to represent a compound quantum channel, we have a bipartite system, and for calculating the entanglement generating capacity of a compound quantum channel, we can use the technique which is similar to the case of a single quantum channel. However, if we use the Stinespring dilation to represent a compound quantum channel, we have a tripartite system which includes the sender, the receiver, and in addition, the environment. Unlike in the case of a single quantum channel, for compound quantum channel we have to deal with uncertainty at the environment. If the sender knows the CSI, the transmitters can build an $(n, \epsilon)$ code for entanglement generating with rate $\min _{t}\left[\chi\left(X ; Q_{t}\right)-\chi\left(X ; E_{t}\right)\right]-\delta=$ $\min _{t \in \theta} I_{C}\left(\rho ; N_{t}\right)-\delta$ (Proposition 1) for any positive $\delta$ and $\epsilon$. This result is optimal (cf. [12]). But if the sender does not know the CSI, he has to build an encoding operator by considering every possible channel 
state for the environment. Therefore the maximal rate that we can achieve is $\min _{t} \chi\left(X ; Q_{t}\right)-\max _{t} \chi\left(X ; E_{t}\right)$, but not $\min _{t \in \theta} I_{C}\left(\rho ; N_{t}\right)=\min _{t}\left[\chi\left(X ; Q_{t}\right)-\chi\left(X ; E_{t}\right)\right]$. This is only a lower bound of the entanglement generating capacity. It is unknown if we can achieve the stronger result (101) using the Stinespring dilation.

\section{ACKNOWLEDGMENT}

Support by the Bundesministerium für Bildung und Forschung (BMBF) via grant 16BQ1050 and 16BQ1052, and the National Natural Science Foundation of China via grant 61271174 is gratefully acknowledged. We would like to thank the reviewers for their valuable comments which helped us to improve our manuscript.
[1] R. Ahlswede, Elimination of correlation in random codes for arbitrarily varying channels, Z. Wahrscheinlichkeitstheorie und verw. Geb., Vol. 44, 159-185, 1978.

[2] R. Ahlswede and V. Blinovsky, Classical capacity of classical-quantum arbitrarily varying channels, IEEE Trans. Inform. Theory, Vol. 53, No. 2, 526-533, 2007.

[3] R. Ahlswede and A. Winter, Strong converse for identification via quantum channels, IEEE Trans. Inform. Theory, Vol. 48, No. 3, 569-579, 2002. Addendum: IEEE Trans. Inform. Theory, Vol. 49, No. 1, 346, 2003.

[4] K. M. R. Audenaert, A sharp continuity estimate for the von Neumann entropy, J. Phys. A: Math. Theor., Vol. 40, 8127-8136, 2007.

[5] H. Barnum, E. Knill, M. A. Nielsen, On Quantum Fidelities and Channel Capacities, IEEE Trans. Inform. Theory, Vol. 46, 1317-1329, 2000.

[6] H. Barnum, M. A. Nielsen, and B. Schumacher, Information transmission through a noisy quantum channel, Phys. Rev. A, Vol. 57, 4153, 1998.

[7] C. H. Bennett, Quantum cryptography using any two non-orthogonal states, Physical Review Letters, Vol. 68, 3121-3124, 1992.

[8] C. H. Bennett and G. Brassard, Quantum cryptography: public key distribution and coin tossing, Proceedings of the IEEE International Conference on Computers, Systems, and Signal Processing, Bangalore, 175, 1984.

[9] I. Bjelaković and H. Boche, Classical capacities of averaged and compound quantum channels. IEEE Trans. Inform. Theory, Vol. 57, No. 7, 3360-3374, 2009.

[10] I. Bjelaković, H. Boche, G. Janßen, and J. Nötzel, Arbitrarily varying and compound classical-quantum channels and a note on quantum zero-error capacities, Information Theory, Combinatorics, and Search Theory, in Memory of Rudolf Ahlswede, H. Aydinian, F. Cicalese, and C. Deppe eds., Vol.7777, 247-283, arXiv:1209.6325, 2012.

[11] I. Bjelaković, H. Boche, and J. Nötzel, Entanglement transmission and generation under channel uncertainty: universal quantum channel coding, Communications in Mathematical Physics, Vol. 292, No. 1, 55-97, 2009.

[12] I. Bjelaković, H. Boche, and J. Nötzel, Entanglement transmission capacity of compound channels, Proc. of International Symposium on Information Theory ISIT 2009, 1889-1893, Korea, 2009.

[13] I. Bjelaković, H. Boche, and J. Sommerfeld, Capacity results for compound wiretap channels, Problems of Infor- mation Transmission, Vol. 49, No. 1, 73-98, 2013; original Russian text: Problemy Peredachi Informatsii, Vol. 49, No. 1, 83-111, 2011.

[14] D. Blackwell, L. Breiman, and A. J. Thomasian, The capacity of a class of channels, Ann. Math. Stat. Vol. 30, No. 4, 1229-1241, 1959.

[15] V. Blinovsky and M. Cai, Classical-quantum arbitrarily varying wiretap channel, Information Theory, Combinatorics, and Search Theory, in Memory of Rudolf Ahlswede, H. Aydinian, F. Cicalese, and C. Deppe eds., Vol.7777, 234-246, 2013.

[16] M. Bloch and J. N. Laneman, On the secrecy capacity of arbitrary wiretap channels, Communication, Control, and Computing, Forty-Sixth Annual Allerton Conference Allerton House, UIUC, USA, 818-825, 2008

[17] H. Boche, M. Cai, and C. Deppe, ClassicalQuantum Arbitrarily Varying Wiretap Channel-A Capacity Formula with Ahlswede Dichotomy-Resources, arXiv:1307.8007, 2013.

[18] M. Cai and N. Cai, Channel state detecting code for compound quantum channel, preprint.

[19] N. Cai, A. Winter, and R. W. Yeung, Quantum privacy and quantum wiretap channels, Problems of Information Transmission, Vol. 40, No. 4, 318-336, 2004.

[20] I. Devetak, The private classical information capacity and quantum information capacity of a quantum channel, IEEE Trans. Inform. Theory, Vol. 51, No. 1, 44-55, 2005

[21] I. Devetak and A. Winter, Distillation of secret key and entanglement from quantum states, Proc. R. Soc. A, Vol. 461, 207-235, 2005.

[22] M. Fannes, A continuity property of the entropy density for spin lattice systems, Communications in Mathematical Physics, Vol. 31. 291-294, 1973.

[23] A. Holevo, Statistical problems in quantum physics, Proceedings of the second Japan-USSR Symposium on Probability Theory, ser. Lecture Notes in Mathematics, G. Maruyama and J. V. Prokhorov, Eds., Vol. 330, 104-119, Springer-Verlag, Berlin, 1973.

[24] A. Holevo, The Capacity of the Quantum Channel with General Signal States, IEEE Trans. on Inf. Theory, Vol. 44, No. 1, 269-273, 1998.

[25] R. Klesse, Approximate quantum error correction, random codes, and quantum channel capacity, Phys. Rev. A 75, 062315, 2007. 
[26] K. Kraus, States, Effects, and Operations, Springer, Berlin, 1983.

[27] D. Leung, J. Lim, and P. Shor, On quantum capacity of erasure channel assisted by back classical communication, Phys. Rev. Lett., Vol 103, No. 24, 240505, 2009.

[28] Y. Liang, G. Kramer, H. Poor, and S. Shamai, Compound wiretap channels, EURASIP Journal on Wireless Communications and Networking - Special issue on wireless physical layer security archive, Vol. 2009, Article No. 5, 2009.

[29] S. Lloyd, Capacity of the noisy quantum channel, Physical Review A, Vol. 55, No. 3, 1613-1622, 1997.

[30] V. D. Milman and G. Schechtman, Asymptotic Theory of Finite Dimensional Normed Spaces. Lecture Notes in Mathematics 1200, Springer-Verlag, corrected second printing, Berlin, UK, 2001.

[31] M. Nielsen and I. Chuang, Quantum Computation and Quantum Information, Cambridge University Press, 2000.

[32] T. Ogawa and H. Nagaoka, Making good codes for classical-quantum channel coding via quantum hypothesis testing, IEEE Trans. Inform. Theory, Vol. 53, No. 6, 2261-2266, 2007.

[33] V. Paulsen, Completely Bounded Maps and Operator Algebras, Cambridge Studies in Advanced Mathematics 78, Cambridge University Press, Cambridge, UK, 2002.
[34] B. Schumacher and M. A. Nielsen, Quantum data processing and error correction, Phys. Rev. A, Vol. 54, 2629, 1996.

[35] B. Schumacher and M. D. Westmoreland, Sending Classical Information via Noisy Quantum Channels, Phys. Rev. A, Vol. 56, No. 1, 131-138, 1997.

[36] B. Schumacher and M. D. Westmoreland, Approximate quantum error correction, Quant. Inf. Proc., Vol. 1, No. 8, 5-12, 2002.

[37] P. W. Shor, The quantum channel capacity and coherent information, lecture notes, MSRI Workshop on Quantum Computation, 2002.

[38] W. F. Stinespring, Positive functions on $C^{*}$-algebras, Proc. Amer. Math. Soc., Vol. 6, 211, 1955.

[39] S. Watanabe, Private and quantum capacities of more capable and less noisy quantum channels, Phys. Rev., A 85, 012326, 2012.

[40] M. Wilde, Quantum Information Theory, Cambridge University Press, 2013.

[41] A. Winter, Coding theorem and strong converse for quantum channels, IEEE Trans. Inform. Theory, Vol. 45, No. 7, 2481-2485, 1999.

[42] A. D. Wyner, The wire-tap channel, Bell System Technical Journal, Vol. 54, No. 8, 1355-1387, 1975. 